\title{
A New Mechanism for Twin Growth in Mg Alloys ${ }^{\dagger}$
}

\author{
A Luque \\ Institute of Mechanical Engineering, École Polytechnique Fédérale de Lausanne. \\ EPFL STI IGM Station 9, CH-1015 Lausanne, Switzerland. Corresponding author. \\ E-mail: aitor.luque@epfl.ch. Phone: +41 216937605.

\section{Ghazisaeidi} \\ Department of Materials Science and Engineering, Ohio State University, 43210 \\ Columbus, OH, USA. E-mail: ghazisaeidi.1@osu.edu

\section{W A Curtin} \\ Institute of Mechanical Engineering, École Polytechnique Fédérale de Lausanne. \\ CH-1015 Lausanne, Switzerland. E-mail: william.curtin@epfl.ch.
}

\begin{abstract}
Twinning is an important deformation mode in lightweight $\mathrm{Mg}$ alloys, but the mechanisms of nucleation and growth of twins and their interactions with solutes remain largely unresolved. Here, a new model for thermally-activated, stress-driven growth of twin boundaries is presented and the role of random and segregated solutes in controlling this growth is studied analytically and using direct molecular dynamics simulations. Twin growth occurs by the thermallyactivated nucleation and expansion of twin dislocation loops on a pre-existing twin boundary. Fluctuations in the local concentration of random solutes lowers the energy barrier for this process and thus facilitates twin growth. Segregation of solutes to the twin boundary strongly increases the energy barrier and suppresses twin growth. In random solid solutions at experimental strain rates, the thermally-activated nucleation process is sufficiently fast at low applied stresses so that growth of the twin is controlled by solute strengthening of the nucleated twin loop as it expands across the twin boundary. Annealing of a deformed sample leads to solute segregation and very strong pinning so that further twinning can only be accomplished by nucleation of new twins, consistent with experimental observations. The new mechanism also (i) operates for detwinning and rationalizes complex twin shapes observed in experiments and (ii) suggests a process for dynamic strain aging commonly observed in $\mathrm{Mg}$ alloys at elevated temperatures.
\end{abstract}

\section{Keywords}

Twinning, Mg alloys, solute fluctuation, segregation, strengthening.

$\dagger$ NOTICE: this is the author's version of a work that was accepted for publication in Acta Materialia. Changes resulting from the publishing process, such as peer review, editing, corrections, structural formatting, and other quality control mechanisms may not be reflected in this document. Changes may have been made to this work since it was submitted for publication. A definitive version was subsequently published in Acta Mat., DOI: 10.1016/j.actamat.2014.08.052. The present text and data are under a CC-BY-NC-ND license. 


\section{Introduction}

The attractiveness of magnesium for structural applications where low weight is required is well-established. However, ductility of $\mathrm{Mg}$ is limited due to its strong plastic anisotropy, in which the activation of slip with components in the $\langle c\rangle$ direction requires very high applied stresses. This drawback is partially mitigated by alloying the pure material and optimizing the processing route and resulting texture in polycrystals [1-3]. Solute elements $(\mathrm{Al}, \mathrm{Zn}, \mathrm{Mn}$, rare-earth elements...) modify texture and critical resolved shear stresses, having a beneficial impact on ductility or formability. Complementing experiments, modeling and simulation studies are progressively providing a deeper understanding of the effects of alloying elements on aspects such as solute-dislocation interactions, interface and grain boundary structures and energies, critical stresses for dislocation motion, dislocation-dislocation interactions and grain-boundary effects [4-10], while texture effects are being understood through crystal plasticity models [11-13].

Twinning and basal slip are the main deformation mechanisms in $\mathrm{Mg}$ and its alloys. There are various twinning systems [14], and here we focus on the so-called tension twin, which is the preferential system activated in a $\mathrm{Mg}$ single crystal loaded in tension along the [0001] direction. The tension twin is associated with the creation and motion of twinning dislocations on the $\{10 \overline{1} 2\}\langle\overline{1} 011\rangle$ system $[15,16]$, with Burgers vector $b_{t w} \sim 0.498 \AA[8]$. The twin dislocation has a step character, with deformation not only on the glide plane but also on neighboring crystallographic planes, with the minimum step height $h \sim 3.86 \AA$, the distance between two $\{10 \overline{1} 2\}$ planes. While the structure is understood, the nucleation and growth of tensile twins, which determines the plastic response of the polycrystalline material at moderate stresses and moderate plastic strains, is not well-established. There are a number of proposed mechanisms for twin nucleation, i.e. the creation of an entirely new twin, that are usually associated with surfaces or defects that provide a local stress concentration to help facilitate the nucleation process. These include homogeneous nucleation from a free surface [17], dislocation-twin interactions [18-21], twin-twin interactions [22], and precipitation/void-induced nucleation $[23,24]$. The shear-coupled migration (SCM) phenomenon for grain boundary motion [25-28] has been extensively studied in terms of interfacial structure (coherency and grain-boundary dislocations or disconnections). New work has studied the solute-strengthening of a pre-existing twin dislocation moving along a pre-existing twin boundary in a material with a random solid solution [29]. The first set of studies do not address how twins can grow (thicken) once created at the local stress concentration, at experimentally applied load levels. The second set of studies address a possible twin growth mechanism, but without any aspects of thermal activation or identification of the role of solutes. The third study on solute strengthening does not address the creation, or source, of the gliding twin dislocations. Thus, the controlling mechanism for twin thickening or twin boundary migration, and the influence of solutes on the growth process, remains unidentified to date.

In this paper, we propose a new model for twin growth, i.e. for the thickening of an existing twin starting from a micro- or nanoscopic twin boundary, due to stress-driven thermally-activated twin migration. The model applies to both pure Mg and solid-solution alloys, and is entirely different from the recent concept of shear-coupled migration. The model predicts that random solutes 
provide a softening effect, i.e. twin growth occurs at lower stresses in the presence of solutes than in pure $\mathrm{Mg}$. The model also predicts that segregation of solutes to the twin boundary strongly inhibits the migration process. Inputs to the model are only the solute/twin-boundary interaction energies, which are computed from first-principles calculations, and a twin dislocation line energy. We validate the analytic model using carefully designed and controlled molecular dynamics simulations on the $\mathrm{Mg}-\mathrm{Al}$ system, for which a validated interatomic potential for the twin structure and energetics is available. Applying the model to solid solution alloys at experimental rates, we predict that the twin-loop nucleation is a necessary mechanism for twinning growth but occurs very rapidly at low stresses, so that the limiting growth stress is controlled by solute strengthening of the expanding loops [29]. After annealing and induced segregation, the model predicts strong pinning of existing twins so that plastic straining by twinning must proceed by the nucleation of new twins, which has recently been demonstrated experimentally [31].

The remainder of this paper is organized as follows. In Section 2, we revisit the $\{10 \overline{1} 2\}\langle\overline{1} 011\rangle$ (tension) twin boundary in $\mathrm{Mg}$ and analyse the solute/twinboundary interaction energies. In Section 3, we develop a model for the nucleation and growth of a twin boundary accounting for random and segregated solutes. In Section 4, we validate the model against MD simulations for both randomly-distributed solutes and solutes segregated to the twin boundary. In Section 5, we discuss the theoretical predictions within the context of established experimental trends. In Section 6, we summarize our main results.

\section{Tension twin boundary in $\mathrm{Mg}-\mathrm{Al}$ alloys}

Twin interfaces are special symmetrical tilt boundaries, characterized by a misorientation angle of $\sim 87^{\circ}[14]$. The interface corresponds to the $\{10 \overline{1} 2\}$ plane, as shown in Fig. 1. The energy of the twin boundary in pure $\mathrm{Mg}$ as obtained by DFT is $\sim 114 \mathrm{~mJ} \mathrm{~m}^{-2}[30]$. Solutes interact with the twin boundary, showing both attractive (binding) and repulsive interactions. Recent first-principles calculations have been used to predict these solute/twin-boundary interaction energies for $\mathrm{Al}$ and $\mathrm{Zn}$ in the tension twin [29], and related computations have been performed for other solutes in other twin boundaries [31]. Fig. 1 shows the structure of the tension twin boundary and eight unique surrounding sites. Also shown are the binding energies of an $\mathrm{Al}$ atom to each of these sites, as computed for the Mg-Al system using DFT [29] and using molecular statics with the Liu et al. Embedded-Atom-Method (EAM) potential [32]. The good agreement between the EAM energies and DFT energies allows us to use the $\mathrm{Mg}-\mathrm{Al}$ system as a test case in molecular dynamics simulations that validate our theory for twin growth, as discussed in the following sections. Fig. 1 also contains the binding energies computed by DFT for Zn [29,31] and Gd [31], which will be used to make predictions for the corresponding alloys with the present model.

Below, we will consider migration of this twin boundary by a nucleation and growth process. Here, we simply note that when the twin boundary has migrated by one step height, the atomic sites on the initial twin boundary become sites off of the final twin boundary, while atomic sites off of the initial twin boundary are incorporated into the final twin boundary. Using the notation of sites shown 


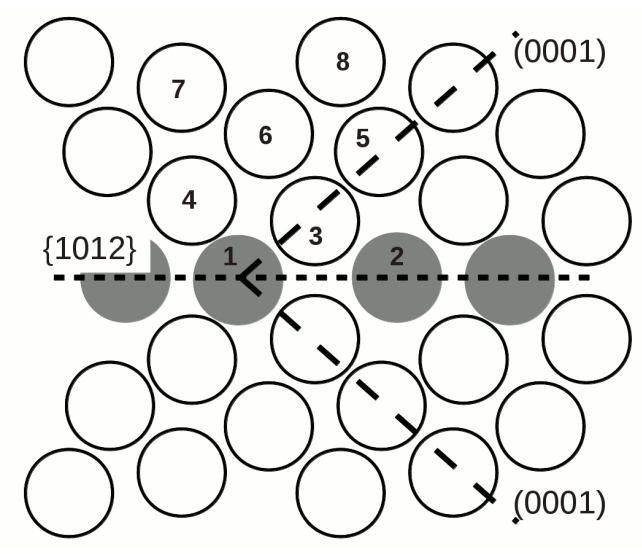

\begin{tabular}{cccccc}
\hline & $\mathrm{Al}$ & $\mathrm{Al}$ & $\mathrm{Zn}$ & $\mathrm{Zn}$ & $\mathrm{Gd}$ \\
Site & $(\mathrm{DFT}[30])$ & $(\mathrm{EAM}[33])$ & $(\mathrm{DFT}[30])$ & $(\mathrm{DFT}[32])$ & $(\mathrm{DFT}[32])$ \\
\hline 1 & -0.150 & -0.129 & -0.220 & -0.265 & +0.197 \\
2 & +0.105 & +0.127 & +0.080 & +0.069 & -0.268 \\
3 & -0.002 & -0.019 & -0.029 & -0.104 & -0.033 \\
4 & -0.023 & -0.045 & -0.045 & -0.104 & -0.060 \\
5 & -0.058 & -0.035 & -0.092 & -0.150 & +0.093 \\
6 & +0.020 & +0.006 & +0.026 & 0 & -0.060 \\
7 & +0.009 & +0.014 & +0.004 & -0.031 & 0 \\
8 & -0.008 & -0.004 & -0.008 & -0.023 & -0.027 \\
\hline
\end{tabular}

Figure 1: Structure of the $\{10 \overline{1} 2\}\langle\overline{1} 011\rangle$ twin boundary and solute/twin boundary interaction energy (in $\mathrm{eV}$ ) for $\mathrm{Al}, \mathrm{Zn}$ and $\mathrm{Gd}$ solutes at various positions along and around a twin boundary in $\mathrm{Mg}$, obtained by DFT or molecular statics.

in Fig. 1, when the twin boundary migrates by one step, all sites 1 become sites 5 and vice-versa, while all sites 2 become sites 6 . In the absence of solutes, there is no difference in energy between the initial and final twin boundaries. In the presence of solutes, there are energy changes associated with the solute distribution on the various sites. As shown in Fig. 1 for $\mathrm{Al}, \mathrm{Zn}$ and $\mathrm{Gd}$, the largest energy changes will occur for solutes lying on sites 1,2,5 and 6 with reference to the initial twin boundary. Such energy differences can provide a driving or retarding force for the twin migration, depending on the statistical distribution of the solutes.

\section{Twin-boundary nucleation model}

\subsection{Model for pure $\mathrm{Mg}$}

Here we are concerned with migration of an initial twin boundary. This initial twin boundary is presumed to have formed due to some unspecified heterogenous nucleation process where a sufficient local stress concentration or local energy concentration exists, such as at a free surface [17] or at a grain boundary $[27,28]$. We consider growth of this initial twin by a nucleation and growth process 
in which an applied stress provides the driving force for the formation of a twinning dislocation loop, i.e. migration of the twin boundary by a unit step over a small region of the boundary bounded by a twinning dislocation loop. If the nucleated twin loop exceeds a critical size, it is unstable to lateral growth across the remaining twin boundary surface. After growth by expansion of the nucleated loop is complete, the entire twin boundary has migrated by one unit step height. Fig. 2 shows schematics of the nucleated state of the system in both an atomistic representation and an idealized representation that will be used to construct our model.

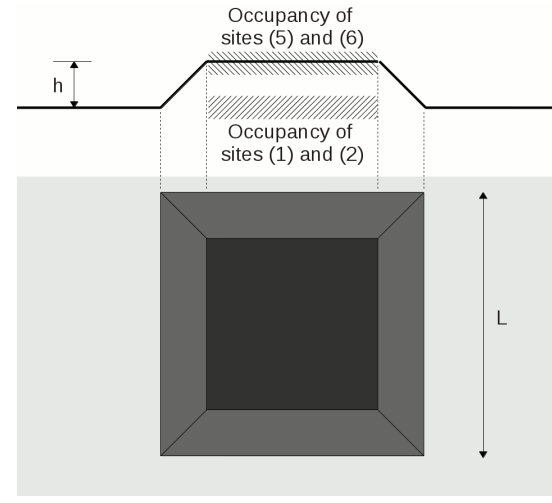

(a)

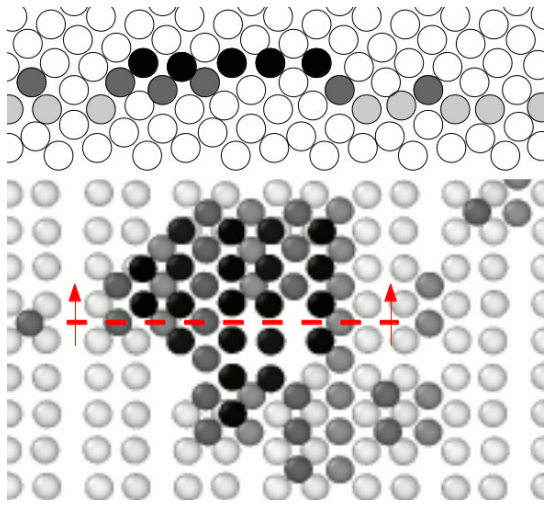

(b)

Figure 2: (a) Schematic configuration of a square twin loop of area $L^{2}$ and height $h$ considered in the model. Bottom: view normal to the twin boundary. Top: cross-section through the twin loop, indicating atomic sites 1, 2, 5 and 6 referenced to the original twin boundary plane. (b) Twin nucleus as observed in a snapshot from an MD simulation. Bottom: view normal to the twin boundary showing only atoms along the twin interface as colored by the atomic $z$ coordinate normal to the twin interface (light gray: atoms in the original twin boundary; black: atoms along twin boundary within the nucleated loop; dark gray: atoms along the twin interface and within the twin that do not occupy perfect twin-lattice positions). Visualization uses OVITO [33]. Top: a crosssection through the nucleated twin nucleus where atoms off the twin interface are shown in white.

The nucleation and growth process follows the classical homogeneous nucleation theory based on an energetic analysis of the system in the initial and nucleated states as a function of the size and shape of the nucleated patch, followed by maximization of the energy versus the size/shape to obtain the energy barrier to nucleation and the associated critical nucleus size. We first describe the process for pure $\mathrm{Mg}$; the inclusion of solute effects follows in the next section. We consider a nucleated loop of square shape with dimensions $L \times L$. The loop perimeter of length $4 L$ consists of the step and twin dislocation necessary to create the twin structure, and we assign an energy cost per unit length denoted by $\Gamma$. Generalizing the analysis to rectangular shape aligned with the edge and screw directions of the twin Burgers vector with dimensions $L_{e d g} \times L_{s c r}$ leads only to a different definition of $\Gamma$ and so we do not need to include this extra 
complication here. The resolved shear stress coupled to the twin Burgers vector $\vec{b}_{t w}$ provides an additional energy term associated with the work done by the applied stress due to the shear deformation caused by the nucleated twin loop. Since the initial and final twin boundaries are identical, there is no change in the surface energy in the pure $\mathrm{Mg}$ case. The energy to create the nucleated loop of size $L \times L$ is thus

$$
\Delta E=4 \Gamma L-\tau b_{t w} L^{2}
$$

The critical nucleated loop size $L^{*}$ is determined by the condition $d \Delta E / d L=0$ at $L=L^{*}$, yielding

$$
L^{*}=2 \Gamma /\left(\tau b_{t w}\right)
$$

and the associated energy barrier to create the critical nucleus is then

$$
\Delta E^{*}=4 \Gamma^{2} /\left(\tau b_{t w}\right)
$$

This critical loop size is unstable to growth, i.e. the total energy decreases as the nucleus grows larger than $L^{*}$, since

$$
\left.\frac{d^{2} \Delta E}{d L^{2}}\right|_{L=L^{*}}=-2 \tau b_{t w}<0
$$

at any non-zero applied stress $\tau$. Growth of the nucleus does require that the applied stress exceed the "Peierls barrier" for twin dislocation motion, but this stress is small in pure Mg. Therefore, the thickening of the twin by one unit $h$ is controlled by this loop nucleation process.

Equations 2 and 3 above show that the critical nucleus size and associated energy barrier decrease with increasing applied stress $\tau$, as expected. At finite temperature, the nucleation barrier can be overcome by thermal activation. If we consider a single initial twin spanning the area of a cubic grain of grain size $D$, then the total initial twin area is $D^{2}$ and there are approximately $N_{t o t}=$ $\left(D / L^{*}\right)^{2}$ possible independent locations for the loop nucleation to occur. We take the attempt frequency for loop nucleation $\nu_{0}^{*}$ to scale inversely with the number of atoms $N$ involved in the nucleation process, $\nu_{0}^{*}=\nu_{0} / N$ where $N=$ $L^{* 2} / \Sigma$ with $\Sigma$ the twin-boundary area per atom and with $\nu_{0}$ an atomic vibration frequency. Thus, the growth rate of the twin in a grain of size $D$ at temperature $T$ and applied stress $\tau$ follows the Arrhenius model given by

$$
R=h \nu_{0}^{*} N_{t o t} \exp \left(-\frac{\Delta E^{*}}{k_{B} T}\right)=h \nu_{0} \frac{\Sigma D^{2}}{L^{*} 4} \exp \left(-\frac{4 \Gamma^{2}}{k_{B} T \tau b_{t w}}\right)
$$

All parameters in the model are material parameters, although precise values for $\Gamma$ and $\nu_{0}$ are difficult to compute and will be determined by comparison of the model to direct MD simulations.

\subsection{Effect of solutes}

In the presence of solutes, whether random or partially/fully segregated to the initial twin boundary, there is an additional energy contribution associated with the difference in surface energy between the initial state (no twin loop) and the nucleated state (with twin loop). In the random-solute case (no segregation), this energy difference is associated with solute concentration fluctuations in 
the two configurations. Since nucleation can occur anywhere on the initial interface, it will occur preferentially in regions where solute fluctuations lower the energy barrier, leading to easier nucleation relative to pure $\mathrm{Mg}$. In the presence of segregation to the initial twin boundary, there is an additional energy cost for nucleating a loop above this boundary that depends on the average segregated solute concentration. Segregation thus stabilizes the initial twin and higher stresses are needed to grow the twin relative to pure $\mathrm{Mg}$. Our analysis here considers the general case of partial segregation, from which all situations ranging from random to full segregation can be obtained. The results for $\mathrm{Al}, \mathrm{Zn}$ and $\mathrm{Gd}$ show that the sites 1, 2, 5, and 6 have the largest solute binding/repulsion energies, and so these sites will dominate the twin nucleation process (Fig. 1). Our analysis will focus on these sites and neglect the (small) effect of sites 3, 4, 7, 8, and all more-distant sites around the twin boundary.

Considering the formation of a twin dislocation loop, as shown in Fig. 2, we denote the number of solutes occupying sites 1 and 5 as $n_{1}$ and $n_{5}$ respectively. Upon formation of the twin loop, the interface moves by one unit of step height and solutes within the loop area in sites 1 become sites of type 5 , and vice versa. The additional energy change due to these solutes is then

$$
\Delta E_{\text {interf }}^{1 \leftrightarrow 5}=\left(n_{1}-n_{5}\right)\left(E_{5}-E_{1}\right)
$$

Similarly, the energy change for solutes in sites 2 and 6 is

$$
\Delta E_{\text {interf }}^{2 \leftrightarrow 6}=\left(n_{2}-n_{6}\right)\left(E_{6}-E_{2}\right)
$$

Differences between $n_{1}$ and $n_{5}$ and between $n_{2}$ and $n_{6}$ arise due to solute segregation and/or random fluctuations. For a loop area $L^{2}$ containing $N=L^{2} / \Sigma$ possible solute sites, the average number of solutes in sites $i, n_{i}$, can be expressed in terms of the site $i$ solute concentration $c_{i}$ as

$$
\bar{n}_{i}=c_{i} N / 2=c_{i} L^{2} /
$$

recognizing that only one-half $(N / 2)$ of the sites are of type $i$. The probability distribution for statistical fluctuations in the number of solutes in sites $i$ in the loop area follows, in the large $N$ limit, a Gaussian distribution with a standard deviation [34]

$$
\Delta n_{i}=\sqrt{c_{i}\left(1-c_{i}\right) N / 2}=L \sqrt{c_{i}\left(1-c_{i}\right) /(2 \Sigma)} .
$$

Fluctuations scale with this standard deviation. We consider a fluctuation of magnitude $\alpha_{i} \Delta n_{i}$, for which the number of solutes in sites $i$ is

$$
n_{i}=\bar{n}_{i}+\alpha_{i} \Delta n_{i}=\frac{c_{i} L^{2}}{2 \Sigma}+\alpha_{i} L \sqrt{\frac{c_{i}\left(1-c_{i}\right)}{2 \Sigma}}
$$

Applying the above results to sites 1 and 5, we obtain the additional energy change upon nucleation of a loop of size $L^{2}$ due to solutes with specified fluctuations in sites 1 and 5 as

$$
\begin{aligned}
\Delta E_{\text {interf }}^{1 \leftrightarrow 5} & =L^{2} \frac{\left(c_{1}-c_{5}\right)}{2 \Sigma}\left(E_{5}-E_{1}\right) \\
& +L\left[\alpha_{1} \sqrt{\frac{c_{1}\left(1-c_{1}\right)}{2 \Sigma}}-\alpha_{5} \sqrt{\frac{c_{5}\left(1-c_{5}\right)}{2 \Sigma}}\right]\left(E_{5}-E_{1}\right)
\end{aligned}
$$


A similar result holds for sites 2 and 6 , i.e.

$$
\begin{aligned}
\Delta E_{\text {interf }}^{2 \leftrightarrow 6} & =L^{2} \frac{\left(c_{2}-c_{6}\right)}{2 \Sigma}\left(E_{6}-E_{2}\right) \\
& +L\left[\alpha_{2} \sqrt{\frac{c_{2}\left(1-c_{2}\right)}{2 \Sigma}}-\alpha_{6} \sqrt{\frac{c_{6}\left(1-c_{6}\right)}{2 \Sigma}}\right]\left(E_{6}-E_{2}\right)
\end{aligned}
$$

The inclusion of these additional energy contributions into the process of loop nucleation and growth is subtle. Segregation is related to the state of the entire twin area whereas fluctuations vary from place to place over the twin area. Therefore, the energy associated with solute segregation must be considered in determining the critical loop size $L^{*}$. In contrast, a local fluctuation over the area of the nucleated loop can facilitate nucleation but cannot be included in the energy minimization because that local fluctuation cannot contribute to the subsequent unstable growth of the loop (the loop will grow, on average, into a surrounding average random environment). The critical loop size $L^{*}$ is thus independent of the fluctuations, but the system will nucleate in regions where the local solute fluctuations lower the energy barrier for nucleation at size $L^{*}$. The energy minimization is thus carried out by including the average solute concentrations only, corresponding to the energy

$$
\Delta E=4 \Gamma L-\tau b_{t w} L^{2}+\tau_{p i n} b_{t w} L^{2}
$$

where we have introduced the stress quantity $\tau_{p i n}$ given by

$$
\tau_{p i n}=\frac{1}{2 b_{t w} \Sigma}\left[\left(c_{1}-c_{5}\right)\left(E_{5}-E_{1}\right)+\left(c_{2}-c_{6}\right)\left(E_{6}-E_{2}\right)\right]
$$

that will emerge as the pinning stress for nucleation in the presence of solute segregation. The minimization then leads to a critical size $L^{*}$ given by

$$
L^{*}=\frac{2 \Gamma}{\left(\tau-\tau_{p i n}\right) b_{t w}}
$$

However, the energy barrier for the nucleation must include the contribution due to the solute fluctuations over the area $L^{* 2}$ of the loop so that

$$
\Delta E^{*}=\frac{4 \Gamma^{2}}{\left(\tau-\tau_{p i n}\right) b_{t w}}+\Delta \Gamma L^{*}
$$

where $\Delta \Gamma$ is an additional positive or negative energy associated with solute fluctuations and given by

$$
\begin{array}{r}
\Delta \Gamma=\frac{E_{5}-E_{1}}{\sqrt{2 \Sigma}}\left[\alpha_{1} \sqrt{c_{1}\left(1-c_{1}\right)}-\alpha_{5} \sqrt{c_{5}\left(1-c_{5}\right)}\right] \\
+\frac{E_{6}-E_{2}}{\sqrt{2 \Sigma}}\left[\alpha_{2} \sqrt{c_{2}\left(1-c_{2}\right)}-\alpha_{6} \sqrt{c_{6}\left(1-c_{6}\right)}\right]
\end{array}
$$

We now address the physically relevant signs (positive and negative) for the terms in the above expressions. Segregation toward thermodynamic equilibrium 
will preferentially populate the low-energy sites. Thus, for $\mathrm{Al}$ and $\mathrm{Zn}$ we expect to have $c_{1}>c_{5}>c_{6}>c_{2}$, and the reverse for Gd. Such segregation will always lower the energy of the initial state, inhibiting nucleation and leading to a positive finite pinning stress $\tau_{\text {pin }}$. Fluctuations along the twin boundary that lower the energy barrier will facilitate nucleation. For Al and $\mathrm{Zn}, E_{5}>E_{1}$ and $E_{6}<E_{2}$ so that lower energy fluctuations occurs for negative fluctuations (depletion) in sites 1 and $6\left(\alpha_{1}, \alpha_{6}<0\right)$, and positive fluctuations (enhancement) in sites 2 and $5\left(\alpha_{2}, \alpha_{5}>0\right)$. For Gd, lower energy fluctuations occur for negative fluctuations in sites 2 and 5 and positive fluctuation in sites 1 and $6\left(\alpha_{2}, \alpha_{5}<0 ; \alpha_{1}, \alpha_{6}>0\right)$. For the remainder of this work, we consider only thermodynamic segregation trends and energy-lowering favorable fluctuations. Introducing the result for $L^{*}$ above and absolute values for the magnitudes of the fluctuations, we therefore obtain our final general expression for the energy barrier as

$$
\Delta E^{*}=\frac{4 \Gamma^{2}-2 \Gamma|\Delta \Gamma|}{\left(\tau-\tau_{p i n}\right) b_{t w}}
$$

with $\tau_{\text {pin }}$ given by Eq. 11,

$$
\tau_{p i n}=\frac{1}{2 b_{t w} \Sigma}\left[\left(c_{1}-c_{5}\right)\left(E_{5}-E_{1}\right)+\left(c_{2}-c_{6}\right)\left(E_{6}-E_{2}\right)\right]
$$

and

$$
\begin{aligned}
|\Delta \Gamma| & =\frac{\left|E_{5}-E_{1}\right|}{\sqrt{2 \Sigma}}\left[\left|\alpha_{1}\right| \sqrt{c_{1}\left(1-c_{1}\right)}+\left|\alpha_{5}\right| \sqrt{c_{5}\left(1-c_{5}\right)}\right] \\
& +\frac{\left|E_{6}-E_{2}\right|}{\sqrt{2 \Sigma}}\left[\left|\alpha_{2}\right| \sqrt{c_{2}\left(1-c_{2}\right)}+\left|\alpha_{6}\right| \sqrt{c_{6}\left(1-c_{6}\right)}\right]
\end{aligned}
$$

valid for $\tau>\tau_{p i n}$ to ensure loop unstable growth condition $\left(d^{2} \Delta E / d L^{2}<0\right.$ at $\left.L=L^{*}\right)$.

The energy barrier in Eq. 15 pertains to a given magnitude of favorable fluctuations, i.e. a set of $\left\{\alpha_{i}\right\}$. Larger fluctuations yield faster growth, and so nucleation and growth of the twin will thus occur at the largest probable value of the $\left\{\alpha_{i}\right\}$ that can be found over the total number of possible nucleation sites $N_{\text {tot }}=\left(D / L^{*}\right)^{2}$. In other words, nucleation and growth is an extreme-value (weak-link) problem and an appropriate statistical analysis is required. The $\left\{\alpha_{i}\right\}$ are independent random variables (fluctuations in sites $i$ are independent of those in sites $j$ ) coming from normalized Gaussian distributions. Thus, the probability of finding a fluctuation $\left|x_{i}\right|$ larger than $\left|\tilde{\alpha}_{i}\right|$ is

$$
p\left(\left|x_{i}\right|>\left|\tilde{\alpha}_{i}\right|\right)=\int_{\left|\tilde{\alpha}_{i}\right|}^{\infty} \frac{d x}{\sqrt{2 \pi}} \exp \left(-\frac{x^{2}}{2}\right)=\int_{-\infty}^{-\left|\tilde{\alpha}_{i}\right|} \frac{d x}{\sqrt{2 \pi}} \exp \left(-\frac{x^{2}}{2}\right)
$$

The median fluctuation $\alpha_{i}$ corresponding to this probability satisfies

$$
\frac{p\left(\left|x_{i}\right|>\left|\alpha_{i}\right|\right)}{p\left(\left|x_{i}\right|>\left|\tilde{\alpha}_{i}\right|\right)}=\frac{1}{2}
$$

The probability of finding a set of fluctuations larger than $\left\{\left|\alpha_{i}\right|\right\}$ for $i=1$, $2,5,6$, is then the product of the individual probabilities of obtaining the corresponding $\left\{\left|\tilde{\alpha}_{i}\right|\right\}$,

$$
P\left(\left\{\alpha_{i}\right\}\right)=\prod_{i=1,2,5,6} p\left(\left|x_{i}\right|>\left|\tilde{\alpha}_{i}\right|\right)=\prod_{i=1,2,5,6} 2 p\left(\left|x_{i}\right|>\left|\alpha_{i}\right|\right)
$$


The largest typical fluctuation that can be found among the $N_{\text {tot }}$ possible nucleation sites then satisfies

$$
P\left(\left\{\alpha_{i}\right\}\right) N_{\text {tot }}=1
$$

The twin nucleation and growth rate is maximized by maximizing the quantity $|\Delta \Gamma|$ (Eq. 17). The maximum twin nucleation rate is thus accurately obtained by maximizing

$$
\begin{aligned}
& \left|E_{5}-E_{1}\right|\left[\left|\alpha_{1}\right| \sqrt{c_{1}\left(1-c_{1}\right)}+\left|\alpha_{5}\right| \sqrt{c_{5}\left(1-c_{5}\right)}\right] \\
& +\left|E_{6}-E_{2}\right|\left[\left|\alpha_{2}\right| \sqrt{c_{2}\left(1-c_{2}\right)}+\left|\alpha_{6}\right| \sqrt{c_{6}\left(1-c_{6}\right)}\right]
\end{aligned}
$$

subject to the condition $P N_{t o t}=1$ above. The derivation of the constrained maximization is shown in Appendix A. For large $N_{\text {tot }}$ (i.e. a large number of possible nucleation sites along the twin boundary) and with the accurate simplification $E_{5}-E_{1} \approx E_{6}-E_{2}$, the analytic result scales as

$$
\left|\alpha_{i}\right| \sim \sqrt{2 \ln \left(\frac{4 N_{t o t}}{\pi^{2}}\right) \frac{c_{i}\left(1-c_{i}\right)}{\sum_{j} c_{j}\left(1-c_{j}\right)}}
$$

In a segregated material, the individual values of the set $\left|\alpha_{i}\right|$ depend on $c_{i}$. In the random solid solution with solute concentration $c, c_{i}=c$, all take on the same value $\left|\alpha_{i}\right|=\alpha$. Detailed calculations show that for typical grain sizes $D=10-100 \mu \mathrm{m}$ and typical values of $L^{*}=10-100 \mathrm{~nm}$ (see below), $N_{t o t}$ ranges between $10^{6}$ and $10^{9}$ and $\alpha$ varies in a narrow range $\sim 2.0-2.5$ due to the exceptionally slow variations of $\sqrt{\ln \left(N_{t o t}\right)}$ with $N_{t o t}$. Thus, in the random alloy we can write an accurate approximation for $\alpha$ as

$$
\alpha \simeq \sqrt{\frac{1}{2} \ln \left(\frac{N_{t o t}}{4 \pi^{2}}\right)}
$$

The statistical analysis above shows that the growth rate of the twin is controlled by the growth rate of the single typical largest fluctuation, corresponding to the values $\left|\alpha_{i}\right|$ (Eq. 23). The general expression for the twin growth rate is thus

$$
R=h \nu_{0} \frac{\Sigma}{L^{* 2}} \exp \left(-\frac{4 \Gamma^{2}-2 \Gamma|\Delta \Gamma|}{k_{B} T\left(\tau-\tau_{p i n}\right) b_{t w}}\right)
$$

with $\tau_{\text {pin }}$ given by Eq. 11 and $|\Delta \Gamma|$ given by Eq. 17 . In the random solute case, when $\alpha$ has a specified value given by Eq. 24 and $\tau_{p i n}=0$, we can write the twin growth rate as

$$
\begin{aligned}
R= & h \nu_{0} \frac{\Sigma}{L^{* 2}} \exp \left(-\frac{4 \Gamma^{2}}{k_{B} T \tau b_{t w}}\right) \\
& \times \exp \left(\frac{2 \Gamma \alpha\left(\left|E_{5}-E_{1}\right|+\left|E_{6}-E_{2}\right|\right)}{k_{B} T \tau b_{t w}} \sqrt{\frac{2 c(1-c)}{\Sigma}}\right)
\end{aligned}
$$

The above results are general and will be analyzed below for random solutes and for solutes with increasing degree of segregation, and will be quantitatively validated by long-time finite-temperature molecular dynamics simulations. 


\section{Model Validation}

\subsection{Simulation details}

To demonstrate the quantitative accuracy of the theory, we perform molecular dynamics simulations in pure $\mathrm{Mg}$ and $\mathrm{Mg}$ with random additions of $\mathrm{Al}$ solutes, for various solute concentrations $c$. Although there are subtle aspects to the simulation, in essence we start with a flat twin boundary at a finite temperature, apply a constant shear stress or shear strain rate, and measure the migration rate of the twin boundary over long times. This procedure is repeated for different solute concentrations, different amounts of solute segregation, and over a range of applied stresses.

We create a rectangular atomic simulation cell with axes $x=\langle\overline{1} 011\rangle, y=$ $\langle\overline{1} 210\rangle=\langle a\rangle$, and $z=\langle 10 \overline{1} 2\rangle$ with reference to the standard hcp unit cell. The cell dimensions are $L_{x}=12.1 \mathrm{~nm}, L_{y}=6.4 \mathrm{~nm}$, and $L_{z}=50.0 \mathrm{~nm}$, and contains a twinned region of thickness $25 \mathrm{~nm}$ that spans the $x-y$ plane and is bounded by two twin boundaries with boundary normal vectors in the $\pm z$ direction. Periodic boundary conditions are applied along all three directions. For this orientation, an applied shear $\tau_{x z}$ will couple to the twin Burgers vector $\vec{b}_{t w}$ and provide the driving force for migration of both twin boundaries. The LAMMPS [35] code and the Mg-Al EAM potential proposed by Liu et al. [32] are used for all simulations. For the random alloys, $\mathrm{Al}$ atoms replace $\mathrm{Mg}$ atoms at random lattice sites to attain the desired concentration $c$.

At finite temperature, the imposition of a well-controlled and stable shear stress is essential for obtaining correct thermally-activated and stress-driven phenomena since artificial fluctuations in the stress will trigger nucleation. This requires careful system preparation, as follows. The entire system is first relaxed at the target temperature using temperature rescaling, and two barostats are used each to eliminate the stresses along $x, y$ and $z$, and the shear stress along $x z$, respectively, in the entire simulation box. $\mathrm{Mg}$ is elastically anisotropic and, for the twin geometry, applied shears in $x z$ generate normal stresses of opposite signs in the two twinned regions. The two barostats deal with this issue acting in the sense of maintaining zero total normal stresses and the target shear stress. The twin boundaries have intrinsic interface stresses that generate finite stresses within the bulk grains proportional to the grain size; this is minimized using a sufficiently large system $z$ dimension. After the above relaxation, we deform the system to the shear strain corresponding to the desired shear stress, and set the barostats to maintain the target stress state during the subsequent simulation. For the small deformations induced in the simulation, the normal stresses in the grains generated by elastic anisotropy are negligible and do not affect to nucleation. To the knowledge of the authors of the present work, this is the only procedure that eliminates a build-up of stresses in the grains as interfaces move, and provides high-fidelity control of the applied shear stress. Furthermore, even if the applied stress is stable, if the applied stress is too large then the first nucleation and growth event injects excess energy into the finite-sized system and this excess energy triggers spurious additional nucleation and growth events that are not associated with thermal activation due to the applied stress alone. When such unstable behavior occurs, the true growth rate cannot be measured. Therefore, we present results only under low stresses where the energy released by a nucleation event is not sufficient to generate additional spurious events 
prior to being damped out. The migration thus proceeds by distinct individual migration events that are separated by significant quiescent periods. To achieve such correct thermal activation and over a sufficient range of growth rates to evaluate the model, we must perform simulations over total times ranging from 100 ps to $125 \mathrm{~ns}$.

\subsection{Pure Magnesium}

Figure 3 shows a typical outcome of our MD simulations for pure $\mathrm{Mg}$. Two aspects are important. First, detailed evaluation of the atomistic configurations demonstrates that the twin migration rate is controlled by a nucleation and growth process, which is the basis of the analytic model. In all cases investigated, a small twin loop is formed from an otherwise flat twin boundary. This twin loop then rapidly spreads laterally across the remaining twin boundary. After this nucleation and growth process, the atomic configuration corresponds to a flat twin boundary that has migrated by height $h$. Second, these events occur at well-separated (stochastic) time intervals. The overall process as observed is thus entirely consistent with our model of stochastic, thermally-activated, uncorrelated twin-loop nucleation and growth events driven by the applied stress and leading to a long-time average twin migration rate. The measured migration rate at $300 \mathrm{~K}$ as a function of the applied shear stress $\tau$ is shown in Fig. 4. Results are shown as the logarithm of the migration rate versus $1 / \tau$, which is the dominant trend predicted by the model (see Eq. 5).

We compare predictions of the model against the outcome of our simulations for pure Mg. The simulations are performed using very small (compared to grain sizes) periodic specimens, and this has an impact on the nucleation and growth. Due to the periodicity, a nucleated twin dislocation loop interacts elastically with its own periodic images, which modifies the energy cost for nucleation. This effect is particularly important when the critical size $L^{*}$ approaches the simulation system size $D$. To account for this effect, we have performed discrete dislocation simulations to calculate the elastic interaction energy of a square loop of size $L$ in a square simulation cell of size $D$, in an elastically isotropic material. The simulations use standard dislocation elasticity theory [14] and lead to an additional interaction energy that is very accurately expressed as

$$
\Delta E_{\text {interact }} \approx-k_{1} D\left(\frac{L}{D}\right)^{4}-k_{2} D\left(\frac{L}{D}\right)^{9}
$$

with $k_{1}=12.2 \mathrm{meV}^{-1}$ and $k_{2}=16.2 \mathrm{meV}^{-1}$ and valid up to $L / D \approx 0.9$. This interaction energy is negative, indicating lower energy, and it therefore facilitates nucleation. This energy must be added to the energy given by Eq. 1, so that the total energy is

$$
\Delta E=4 \Gamma L^{2}-\tau b_{t w} L-k_{1} D\left(\frac{L}{D}\right)^{4}-k_{2} D\left(\frac{L}{D}\right)^{9}
$$

Maximization of the new total energy predicts the critical loop size $L^{*}$ and energy barrier $\Delta E^{*}$ for the given periodic simulation. The maximization cannot be performed analytically, however. To perform the maximization numerically requires values for all the parameters, but $\Gamma$ is not known a priori. Therefore, we must proceed numerically and self-consistently to derive the value of $\Gamma$ from 


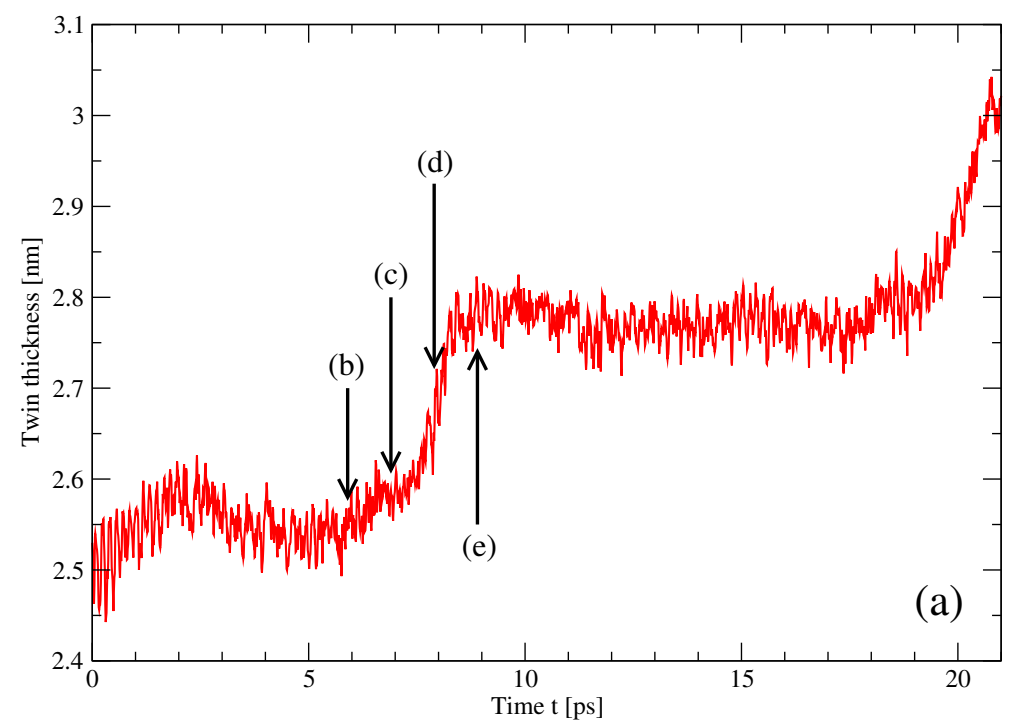

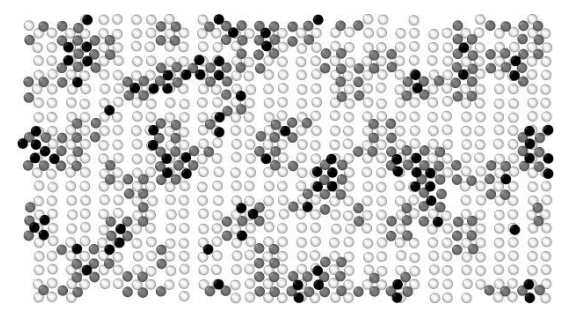

(b)

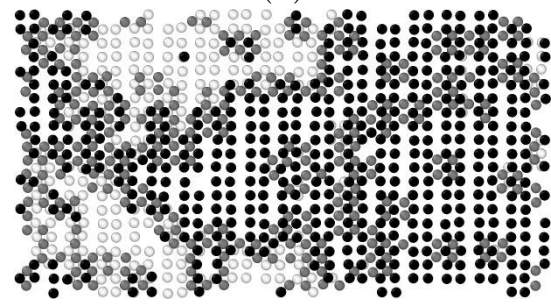

(d)

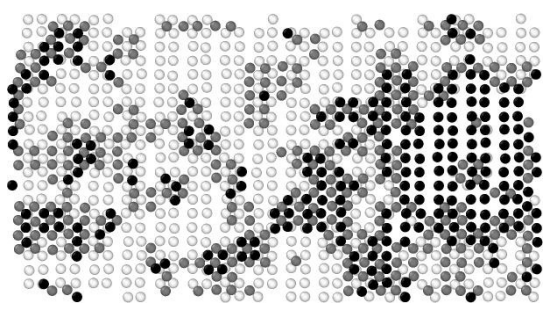

(c)

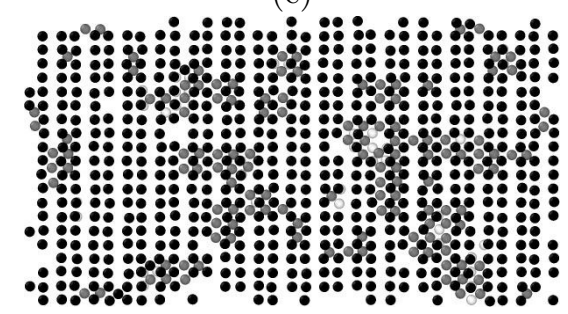

(e)

Figure 3: (a) Twin thickness vs time as measured during one simulation of pure $\mathrm{Mg}(T=300 \mathrm{~K}$ and $\tau=110 \mathrm{MPa})$, showing twin-boundary migration taking place by individual events that occur stochastically, are completed in a short period, and are separated by longer quiescent periods. (b)-(e) Atomistic evolution of the twin boundary during a single nucleation event, demonstrating that twin growth occurs by the localized nucleation of a twin loop that then expands across the remaining boundary, and ending with the system having a new, flat twin boundary. Only twin boundary atoms are shown as colored according to the atomic $z$ coordinate normal to the twin interface (light gray: atoms in the original twin boundary; black: atoms in the nucleated twin boundary; dark gray: atoms around and in the nucleated twin that do not (yet) occupy twin-lattice positions). 


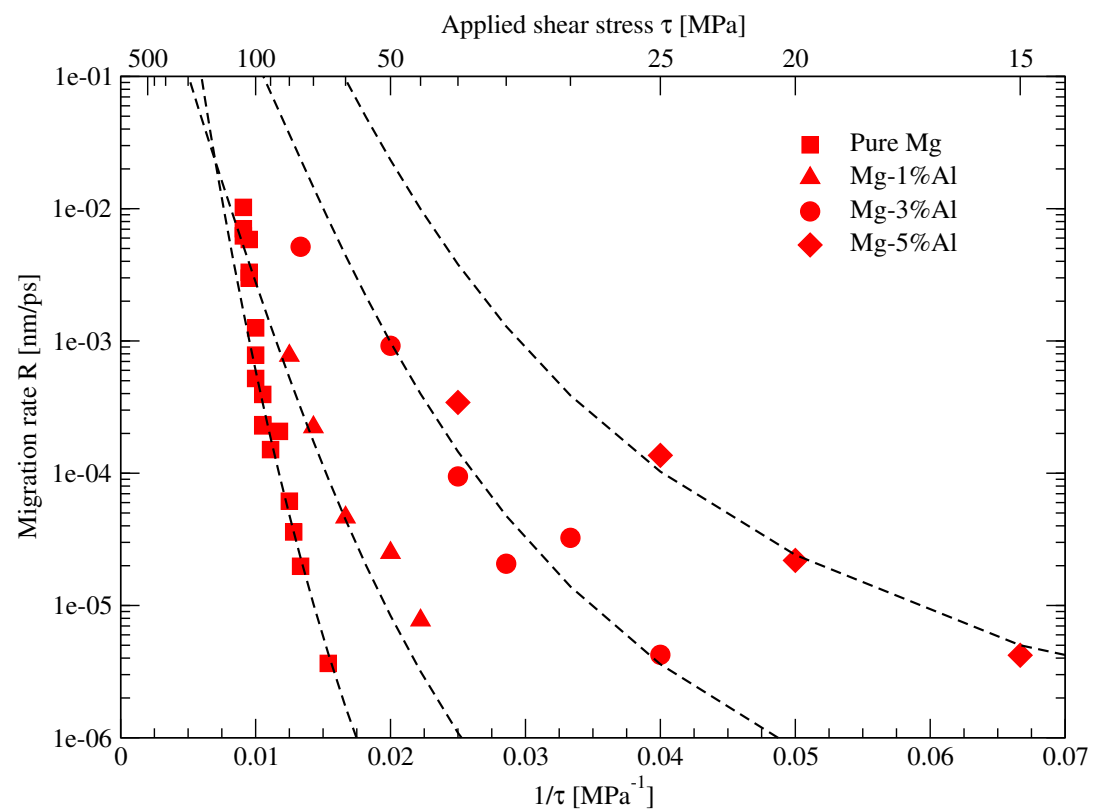

Figure 4: Logarithm of the migration rate $R$ versus inverse of the stress $1 / \tau$, for pure $\mathrm{Mg}$ (squares), $\mathrm{Mg}-1 \% \mathrm{Al}$ (triangles), $\mathrm{Mg}-3 \% \mathrm{Al}$ (circles), $\mathrm{Mg}-5 \% \mathrm{Al}$ (diamonds), at $T=300 \mathrm{~K}$. Solute concentrations are in atomic percentage and refer to random solute distributions. Dashed lines show predictions of the model, with parameters $\Gamma$ and $\nu_{0}$ fitted to the pure $\mathrm{Mg}$ case only.

the twin nucleation rates measured in the simulations on pure Mg. Specifically, we choose a value for the parameter $\Gamma$ and a specified stress $\tau$ and numerically maximize the total energy given by Eq. 28 to obtain $L^{*}$ and $E^{*}$. With these quantities and a value of $\nu_{0}$, and noting that $N_{t o t}=2\left(D / L^{*}\right)^{2}$ because the simulations contain two twin boundaries of area $D^{2}$, we compute the twin growth rate according to Eq. 5. This is repeated as a function of the applied stress $\tau$. We then vary the value of $\Gamma$ (and $\nu_{0}$ ) to fit all the simulation data on pure $\mathrm{Mg}$ versus applied stress. Fig. 4 shows the prediction of our model (including the periodic interactions) with the MD simulations as a function of $1 / \tau$ at $T=300 \mathrm{~K}$ in pure $\mathrm{Mg}$ using the parameters $\Gamma=4.23 \mathrm{meV}^{-1}$ and $\nu_{0}=$ $1.7 \times 10^{13} \mathrm{~s}^{-1}$. The agreement is excellent over several orders of magnitude in the migration rate. The model predicts nucleation loop sizes $L^{*}$ in the range from 25 to $40 \AA$ between $100 \mathrm{MPa}$ and $50 \mathrm{MPa}$, and the inclusion of the periodic image interactions becomes important as the stress decreases below $90 \mathrm{MPa}$. These results simultaneously demonstrate that the model can quantitatively explain the simulation results in pure $\mathrm{Mg}$ and provides values for the only two parameters in the model, which will be held fixed in all subsequent predictions.

\subsection{Random Al solutes in $\mathrm{Mg}$}

Figure 4 shows the twin growth rates measured in the MD simulations as a function of $1 / \tau$ at $T=300 \mathrm{~K}$ in $\mathrm{Mg}$ alloys with $c=1,3$ and 5 at.\% Al. 
The introduction of solutes accelerates the twin nucleation and growth rate, and increasing solute concentrations lead to rapidly increasing growth rate at any given stress. Alternatively, any given growth rate $\mathrm{R}$ can be achieved at a significantly lower stress with increasing solute concentration, with measurable MD growth rates achieved at an applied stress of only $15 \mathrm{MPa}$ for $5 \% \mathrm{Al}$. The simulations thus generally support the trend of the model, in which random solutes can significantly enhance the twin growth rate by reduction of the energy barriers needed for nucleation.

Turning to the model predictions, when the solutes are randomly distributed in the material, with no preference for any site, the concentrations of sites $i$ are identical, i.e., $c_{1}=c_{2}=c_{5}=c_{6}=c$, where $c$ is the average solute concentration in the material. The average number of solutes in sites 1 and 5 , and in sites 2 and 6 are then equal, and the energy contributions due to the average concentrations cancel out. In this situation, $\tau_{\text {pin }}=0$ and

$$
|\Delta \Gamma|=\left[\left(\left|\alpha_{1}\right|+\left|\alpha_{5}\right|\right)\left|E_{5}-E_{1}\right|+\left(\left|\alpha_{2}\right|+\left|\alpha_{6}\right|\right)\left|E_{6}-E_{2}\right|\right] \sqrt{\frac{c(1-c)}{2 \Sigma}}
$$

The difference between pure $\mathrm{Mg}$ and $\mathrm{Mg}$ with random solutes is thus entirely determined by the solute concentration fluctuations and solute/twin-boundary interaction energies with no adjustable parameters.

Because the solutes reduce the magnitude of the energy barrier, nucleation and growth on MD-accessible time scales can be achieved at lower stresses. Lower stresses correspond to larger critical nuclei $L^{*}$, and thus finite-size effects due to twin loop image interactions have even greater importance in interpreting the simulation results. The use of finite-size simulations also has another effect: the number of possible nucleation sites $N_{\text {tot }}$ is limited to a small value, as compared to the analysis developed for realisitic material scales. Thus, the large-fluctuation limit and asymptotic values for $\left|\alpha_{i}\right|=\alpha$ for all $i$ (see Eq. 24 ) is not attainable in the simulations and thus not valid for interpreting the simulations although the general model still applies. The results for pure $\mathrm{Mg}$ determine $L^{*}$ and $N_{t o t}=2\left(D / L^{*}\right)^{2}$ as a function of stress, independently of the solutes. The typical probable favorable fluctuations driving nucleation in the finite-size simulation remain governed by the condition $P\left(\left\{\alpha_{i}\right\}\right) N_{t o t}=1$ and by the maximization of $\sum_{i}\left|\alpha_{i}\right|$ that maximizes the growth rate. Noting that $\alpha_{1}$ and $\alpha_{5}$ are interchangeable, as are $\alpha_{2}$ and $\alpha_{6}$, and as noted earlier that the energy differences are comparable, $\left|E_{5}-E_{1}\right| \approx\left|E_{6}-E_{2}\right|$, we can simplify the analysis by considering an approximate $\Delta \Gamma$ given by

$$
|\Delta \Gamma| \simeq\left(\left|\alpha_{1}\right|+\left|\alpha_{5}\right|+\left|\alpha_{2}\right|+\left|\alpha_{6}\right|\right) \frac{\left|E_{5}-E_{1}\right|+\left|E_{6}-E_{2}\right|}{2} \sqrt{\frac{c(1-c)}{2 \Sigma}}
$$

Since fluctuations on the different sites $i$ are independent, we then evaluate the combinations $\left(\alpha_{1}, \alpha_{2}, \alpha_{5}, \alpha_{6}\right)$ in which (i) all four values of $\alpha$ are equal and nonzero; (ii) three values of $\alpha$ are equal and non-zero and one value is zero; (iii) two values of $\alpha$ are equal and non-zero and two other values are zero; and (iv) one value of $\alpha$ is non-zero and the three remaining values are zero. The value of $\alpha$ is uniquely determined by the condition $P\left(\left\{\alpha_{i}\right\}\right) N_{\text {tot }}=1$ at any given stress. Analysis shows that the condition $P\left(\left\{\alpha_{i}\right\}\right) N_{\text {tot }}=1$ cannot be satisfied with either four or three non-zero values of $\alpha$; in other words it is not statistically possible to find favorable (above-average) fluctuations on any three or four types 
of sites 1, 2, 5 and 6 , for our simulation system sizes and stress levels. We find that all of our simulations can be well-matched with two non-zero values of $\alpha$ at all stress levels; in other words, nucleation in the simulations is controlled by solute fluctuations on two of the four possible types of sites. Situations with one non-zero value of $\alpha$ are statistically possible but do not predict the observed growth rates. Figure 4 shows the model predictions for the growth rate $R$ versus $1 / \tau$, using the values of $\Gamma$ and $\nu_{0}$ obtained for pure $\mathrm{Mg}$, the image interaction energies that determine $L^{*}$, and the value of $\alpha$ satisfying $P\left(\left\{\alpha_{i}\right\}\right) N_{\text {tot }}=1$; there are no fitting parameters in this comparison between theory and simulation. The fully analytic theory provides an excellent description of the simulations when all subtle aspects associated with the finite simulation size are included, and with no adjustable parameters. Solute-fluctuation-driven twin-boundary nucleation in $\mathrm{Mg}$ alloys with random solutes is thus validated as a new mechanism for twin nucleation and growth.

\subsection{Segregated Mg-Al alloys}

Under the combined effect of time and temperature, solutes can diffuse and segregate to atomic sites where the solutes are most strongly bound. For the $\mathrm{Mg}$ twin boundary and $\mathrm{Al}$ or Zn solutes, the energies in Fig. 1 show that site 1 is strongly favorable for segregation, site 5 just off the twin boundary can have weaker segregation, and sites 2 and 6 would be depleted. In Gd, site 2 is strongly favorable for segregation, site 6 is weakly favorable for segregation, and sites 1 and 5 would be depleted. Segregation to binding locations then acts to pin the twin boundary and inhibits twin nucleation and growth. Within simple solution theory [36], the equilibrium concentration of solutes in sites $i$ depends on the overall matrix solute concentration $c$ and temperature $T$, which establishes the solute chemical potential, and the binding energy $E_{i}$, and is given by

$$
c_{i}=\frac{c \exp \left(-E_{i} /\left(k_{B} T\right)\right)}{1+c \exp \left(-E_{i} /\left(k_{B} T\right)\right)}
$$

Since the average solute concentrations on different sites are no longer equal, the energy contributions associated with the average solute concentrations no longer cancel out and, therefore, there is a non-zero "pinning stress" $\tau_{\text {pin }}$ that sets a lower limit to the stresses required to grow the twin away from the segregated boundary.

To test the analytic theory on $\mathrm{Mg}-\mathrm{Al}$, we consider a simplified situation in which there is partial segregation only to sites 1 , and complete depletion of sites 2,5 , and $6\left(c_{2}=c_{5}=c_{6}=0\right)$. In this case,

$$
\tau_{\text {pin }}=c_{1}\left(E_{5}-E_{1}\right) /\left(2 b_{t w} \Sigma\right)
$$

and

$$
|\Delta \Gamma|=\left|\alpha_{1}\right|\left|E_{5}-E_{1}\right| \sqrt{\frac{c_{1}\left(1-c_{1}\right)}{2 \Sigma}}
$$

with $\left|\alpha_{1}\right|$ given by the condition $2 p\left(\left|x_{1}\right|>\left|\alpha_{1}\right|\right) N_{\text {tot }}=1$ since there are no fluctuations associated with other sites. Thermally-activated nucleation is only possible for $\tau>\tau_{\text {pin }}$. Molecular dynamics simulations were performed at a constant shear strain rate $\dot{\gamma} \sim 5 \times 10^{7} \mathrm{~s}^{-1}$ such that the applied shear stress increases monotonically with time, and at $T=300 \mathrm{~K}$. We use the same general 
geometry as discussed earlier, but with dimensions $12.1 \times 6.4 \times 16.0 \mathrm{~nm}^{3}$. We consider site 1 occupancies $c_{1}$ ranging from $10 \%$ to $100 \%$, the latter corresponding to full segregation. We measure the stress at which nucleation and growth occurs as a function of $c_{1}$. Because segregation only pins the initial twin boundary, after unpinning the new twin interface contains no solutes and reverts to the rate of twin growth in pure Mg. Thus, we focus only on the unpinning event.

Starting from zero initial strain, the system deforms elastically with no nucleation possible until the stress $\tau_{p i n}$ is reached. With further straining, $\tau>\tau_{\text {pin }}$, and thermally-activated nucleation becomes possible. To nucleate the twin boundary, and migrate by one unit step, requires an average time that satisfies

$$
\int_{t\left(\tau_{\text {pin }}\right)}^{t(\tau)} R(\tau(t)) d t=h
$$

Time and stress are related through the shear strain rate, $\tau=\mu \dot{\gamma} t$, where $\mu \sim 17$ $\mathrm{GPa}$ is the shear modulus, and hence the average stress at unpinning satisfies

$$
\int_{\tau_{p i n}}^{\tau} \frac{\nu_{0}}{\dot{\gamma}} \frac{\Sigma}{L^{* 2}} \exp \left(-\frac{\Delta E^{*}}{k_{B} T}\right) \frac{d \tau}{\mu}=1
$$

where $L^{*}$ depends on $\tau-\tau_{\text {pin }}$ and where $\Delta E^{*}$, which also depends on $L^{*}$, contains the loop image interaction energy term relevant to the simulations. The integral equation cannot be solved analytically but the unpinning shear stress can be computed numerically.

Fig. 5 shows the unpinning stresses measured in the MD simulations versus the unpinning stresses predicted using the model, with no fitting parameters. The agreement between simulation and theory is excellent; differences can be due to solute-solute interactions at high concentrations that are not considered in the model ${ }^{\ddagger}$. Most importantly, the pinning stresses due to even modest segregation $\left(c_{1}=0.1\right)$ of a relatively low-binding-energy solute $(\mathrm{Al})$ are very high, more than $150 \mathrm{MPa}$.

\section{Predictions and implications for experiments}

We now predict the stresses necessary to achieve twin plastic strain rates in the range of experimentally used deformation rates, assuming that the controlling mechanism is the twin nucleation process studied here. In general, the (plastic) shear strain rate due to twinning, $\dot{\gamma}$, can be related to the nucleation rate as follows. We consider the twinning to occur in cubic grains with grain size $D$. The plastic shear strain rate is the rate of twin growth divided by the grain size and multiplied by the plastic shear strain for twinning,

$$
\dot{\gamma}=R \gamma_{t w} / D
$$

where $\gamma_{t w}=b_{t w} / h \sim 0.13$ is the plastic shear strain associated with one twin dislocation.

\footnotetext{
${ }^{\ddagger}$ By means of MD simulations not presented here, interactions among solutes in sites 1 in a fully-segregated configuration reduce the solute/twin interaction energies associated with these sites from $94 \mathrm{meV}$ to $77 \mathrm{meV}$, which reduces the stresses required for nucleation due to changes in both $\tau_{\text {pin }}$ and $\Delta \Gamma$ (Eqs. 32 and 33, respectively)
} 


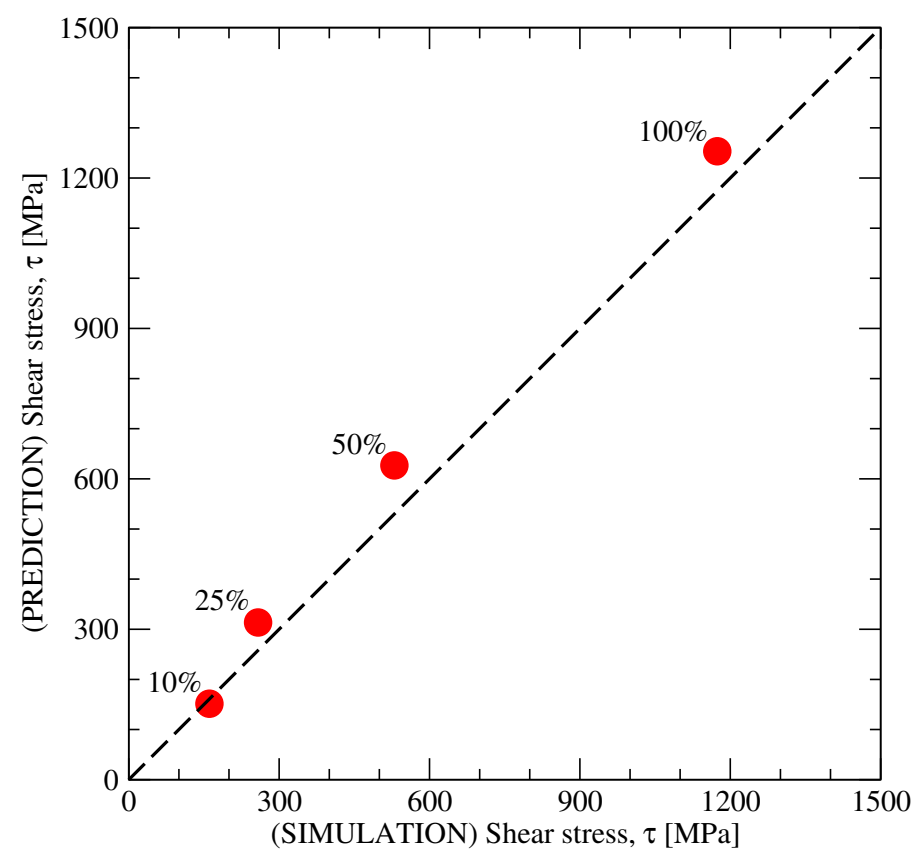

Figure 5: Applied shear stress for unpinning of the twin boundary with solutes segregated to site 1 under application of a constant applied strain rate as measured in the simulation versus the stress predicted by the model, for segregated concentrations $c_{1}=10 \%, 25 \%, 50 \%$ and $100 \%$.

\subsection{Random solid solution alloys}

In the random solute case, $c_{i}=c$, for $i=1,2,5$ and 6 , and $\tau_{\text {pin }}=0$, we can insert $R$ from Eq. 26 into the above equation to give

$$
\dot{\gamma}=\nu_{0} \frac{b_{t w} \Sigma}{D L^{* 2}} \exp \left(-\frac{4 \Gamma^{2}}{k_{B} T \tau b_{t w}}\right) \exp \left(\frac{2 \Gamma|\Delta \Gamma|}{k_{B} T \tau b_{t w}}\right)
$$

Inverting this expression to solve for the flow stress $\tau$ yields

$$
\tau=\frac{2 \Gamma(2 \Gamma-|\Delta \Gamma|)}{k_{B} T b_{t w}}\left[\ln \left(\frac{\nu_{0}}{\dot{\gamma}} \frac{b_{t w} \Sigma}{D L^{* 2}}\right)\right]^{-1}
$$

where

$$
|\Delta \Gamma|=\alpha\left(\left|E_{5}-E_{1}\right|+\left|E_{6}-E_{2}\right|\right) \sqrt{2 c(1-c) / \Sigma}
$$

Strictly, $\alpha$ depends on $L^{*}$, since

$$
\alpha \simeq \sqrt{\frac{1}{2} \ln \left(\frac{N_{t o t}}{4 \pi^{2}}\right)}
$$

and $N_{t o t}=\left(D / L^{*}\right)^{2}$, and $L^{*}$ depends on $\tau$, since $L^{*}=2 \Gamma /\left(\tau b_{t w}\right)$, so that the equation above should be solved self-consistently, but the additional $\tau$ dependencies only appears in slowly-varying logarithmic term and have a very small effect on the final results. 
A crucial feature of Eq. 37 is that if the solute concentration and/or $D$ (and hence $N_{\text {tot }}$ and $\alpha$ ) are sufficiently large then the stress for nucleation can approach zero. The logarithmic term with the full $\tau$ dependence prevents the stress from reaching zero, but the stress can be negligibly small. In this limit, an initially flat interface will spontaneously migrate up and down to form various twin loops across the entire surface. We have observed this phenomenon in our small size MD simulations under zero applied stress at concentrations of $\mathrm{Al}$ exceeding $10 \%$ and, occasionally, at $5 \%$ (results not shown here). The solute concentration necessary to reach this limit can be estimated by setting $2 \Gamma-$ $|\Delta \Gamma|=0$, leading to

$$
c \simeq\left(\frac{\Gamma \sqrt{2 \Sigma}}{\alpha\left(\left|E_{5}-E_{1}\right|+\left|E_{6}-E_{2}\right|\right)}\right)^{2}
$$

In materials with typical large grain sizes $D=10-100 \mu \mathrm{m}$, the controlling fluctuations have $\alpha \sim 2.0-2.5$, and the model predicts spontaneous nucleation at estimated solute concentrations of $\sim 0.0022$ for $\mathrm{Al}, \sim 0.0021$ for $\mathrm{Zn}$, and $\sim 0.0007$ for Gd. Therefore, we conclude that twinning in typical alloys $(c \sim$ 1at.\% or more) is not limited by the nucleation mechanism studied here; the stresses for nucleation can be negligibly small. Thus, we conclude that the observed twin stresses measured in Mg alloys are controlled by either (i) the nucleation of the initial micro or nanotwin that we assume to pre-exist or (ii) by solute strengthening that impedes expansion of the nucleation twin loops. Nonetheless, the present model provides a mechanism by which twin dislocation loops can be nucleated, leading to plastic strain by twinning due to expansion of the nucleated loops.

The solute strengthening of twin dislocations, i.e. the resistance to twin dislocation motion due to the presence of solutes in and around the twin boundary, has recently been studied in depth [29]. That work derives an analytic expression for the solute strengthening given by

$$
\tau=2.4375 c \frac{E_{n}^{3 / 2} \Gamma_{L}^{1 / 2}}{k_{B} T b_{t w}}\left[\ln \left(\frac{\dot{\gamma}_{0}}{\dot{\gamma}}\right)\right]^{-1}
$$

Here, $E_{n}$ is a characteristic energy associated with the collective effect of random solutes pinning the twin dislocation, with $9 \mathrm{meV} \AA$ and $14 \mathrm{meV} \AA$ for $\mathrm{Mg}-\mathrm{Al}$ and $\mathrm{Mg}-\mathrm{Zn}$, respectively [29]. The quantity $\Gamma_{L}$ is an effective line/step energy for the twin dislocation bowing which was computed as $\Gamma_{L} \sim 40 \mathrm{meV} \AA$, and $\dot{\gamma}_{0} \sim 10^{5}$ $\mathrm{s}^{-1}$ is a reference shear strain rate. The similarity of the forms of Eqs. 37 and 39 is interesting, in spite of the quite different physical problems underlying the resulting stress versus strain rate. One major difference between these models, however, is the scaling with concentration: twin dislocation glide is hindered by the presence of solute, with $\tau \propto c$, while twin loop nucleation rate is enhanced by random solutes so that the stress to achieve nucleation at a given rate is decreasing with concentration.

For plastic strain by twinning, the larger of the two values of $\tau$ obtained from Eqs. 37 and 39 will dictate the controlling mechanism. As anticipated from above, the twin nucleation process controls twin plasticity only at very low solute concentrations $(c<0.003$ for $\mathrm{Al}$ or $\mathrm{Zn}$, and $c<0.001$ for $\mathrm{Gd})$. At higher concentrations in the range of industrial alloys, twin growth is controlled by 
the solute strengthening of twin dislocation glide, with the twin loop nucleation occuring very easily at these stress levels. Ghazisaeidi et al. show that the predictions for solute strengthening of twin glide are consistent with measured twin strengths across a range of $\mathrm{AZ}(\mathrm{Mg}-\mathrm{Al}-\mathrm{Zn})$ alloy compositions, indicating that twin dislocation motion is indeed the controlling factor. Thus, the present model provides the physical and necessary mechanism for nucleation of twin loops, which is a prerequisite for attaining subsequent plastic strain by growth of the nucleated twin loops, but the stress for twin plasticity is controlled by the solute strengthening.

\subsection{Pure Mg}

We now step back to consider perfectly pure Mg. As seen in simulations, the twin loop nucleation process is much slower than in the alloy material. Inverting Eq. 5, the stress versus twin plastic strain rate is predicted to be

$$
\tau=\frac{4 \Gamma^{2}}{k_{B} T b_{t w}}\left[\ln \left(\frac{\nu_{0}}{\dot{\gamma}} \frac{b_{t w} \Sigma D}{L^{* 4}}\right)\right]^{-1}
$$

For grain sizes $D=10-100 \mu \mathrm{m}$ and at a typical experimental strain rate of $10^{-3} \mathrm{~s}^{-1}$, Eq. 40 predicts stresses of $\sim 25 \mathrm{MPa}$. Such stresses are rather larger than the widely-accepted value of $3 \mathrm{MPa}$ for twinning in pure $\mathrm{Mg}$ single crystals [37]. Thus, the theory apparently predicts that twinning in pure $\mathrm{Mg}$ should be controlled by nucleation, and at stresses larger than observed experimentally. We address this disagreement below.

The strength results reported to date consider pure $\mathrm{Mg}$ single-crystalline samples with (Ca, Cu, Mn, Ni, Pb, Si, Sn...) impurity contents ranging from $0.03 \%$ [37] to $0.01 \%$ [38], or $0.05 \%$ in pure $\mathrm{Mg}$ polycrystalline samples of grain size ranging from 35 to $200 \mu \mathrm{m}$ [39]. These substitutional impurities play the same role as solutes in our theory, so that substitutional solutes, even at low impurity concentrations, could facilitate the twin nucleation process. As an example, we compute the level of impurities necessary for loop nucleation at the experimentally-measured stress of $3 \mathrm{MPa}$ and a strain rate of $10^{-3} \mathrm{~s}^{-1}$ in otherwise pure $\mathrm{Mg}$ single crystal [37]. We use a grain size of $D \sim 10 \mathrm{~mm}$, corresponding to the specimen size, and an average impurity/twin interaction energy equal to that of Zn. Including the full dependence of $L^{*}$ on $\tau$, we solve Eq. 37 for the required impurity concentration, yielding $c \sim 0.14 \% \S$. We conclude that $\mathrm{Mg}$ specimens of commercial purity $\lesssim 99.86 \%$ would permit nucleation and growth of a twin loop at the experimentally-observed stress for "pure" $\mathrm{Mg}$ due to the impurities. Stronger impurity/twin interaction energies would lower the impurity concentration needed for nucleation at the observed stress. Thus, impurity effects can bring our predictions for "pure" $\mathrm{Mg}$ from $25 \mathrm{MPa}$ into the range of experimental values, but only very approximately. Our estimate above is unable to explain the measured $3 \mathrm{MPa}$ twinning stress in $99.97 \%$ pure $\mathrm{Mg}$, and we do not consider the model to be quantitatively successful for nominally pure Mg.

\footnotetext{
§The critical loop size is $L^{*} \sim 90 \mathrm{~nm}$, the number of nucleation sites is $N_{t o t} \sim 1.2 \times 10^{10}$ and, therefore, $\alpha \sim 3$. At $c=0.0014$, the loop area $L^{* 2}$ contains 190 impurity atoms on average, with $\sim 47$ impurities on average in each of sites 1, 2, 5 and 6 . The necessary fluctuation to cause the loop nucleation would therefore correspond to depletion/enhancement of $\sim 21$ impurities on each of the sites 1, 2, 5 and 6 (see Eq. 8)
} 


\subsection{Alloys with twin-boundary segregation}

We now predict the stress to activate twin nucleation on a segregated twin boundary. As mentioned before, under these circumstances, $\tau \gtrsim \tau_{\text {pin }}$ to produce unpinning, and the thermal activation is relatively unimportant if $\tau_{p i n}$ is large. For equilibrium segregation, we can thus take $\tau \simeq \tau_{\text {pin }}$. We consider that only low-energy sites (sites 1 and 5 in $\mathrm{Al}$ and $\mathrm{Zn}$; sites 2 and 6 in $\mathrm{Gd}$ ) have appreciable segregation. Therefore, segregation predominantly affects two layers of atoms (those containing sites 1 and 2, and those containing sites 5 and 6 ; Fig. 1). The unpinning process can take place in two steps. The twin boundary first has to move from the initial boundary layer containing sites 1 and 2 to the position containing sites 5 and 6 . The twin boundary then moves from this new position, where the former sites 5 and 6 are now sites 1 and 2, into the next layer, which contains no segregation and thus behaves like the random alloy. The operative pinning stress is the larger of the stresses for these two steps, and so, according to Eq. 11, the larger of $c_{1}-c_{5}$ (1st step) and $c_{5}-c$ (2nd step) determines the unpinning stress for $\mathrm{Al}$ and $\mathrm{Zn}$ while the larger of $c_{2}-c_{6}$ (1st step) and $c_{6}-c$ (2nd step) determines the unpinning stress of Gd. Inserting the specific solute binding energies, the theory predicts

$$
\begin{array}{ll}
\tau_{\text {pin }}=(1230 \mathrm{MPa}) \max \left\{c_{1}-c_{5}, c_{5}-c\right\} & \text { for } \mathrm{Mg}-\mathrm{Al} \\
\tau_{\text {pin }}=(1710 \mathrm{MPa}) \max \left\{c_{1}-c_{5}, c_{5}-c\right\} & \text { for } \mathrm{Mg}-\mathrm{Zn} \\
\tau_{\text {pin }}=(2770 \mathrm{MPa}) \max \left\{c_{2}-c_{6}, c_{6}-c\right\} & \text { for } \mathrm{Mg}-\mathrm{Gd}
\end{array}
$$

Because the coefficients are large in all cases, the stress required for unpinning the twin is very high, easily exceeding $100 \mathrm{MPa}$, even for relatively modest $10 \%$ segregation to the boundary. Growth of a twin to which solutes have segregated is thus unlikely at experimental stress levels.

Recent work by Nie et al. is consistent with our conclusion [31]. Nie et al. studied Mg-0.2 at.\% Gd in the following cases: (i) compression to a strain of 0.025 , unloading, and reloading to a total strain of 0.045 ; (ii) compression to 0.025 , unloading, annealing at $150{ }^{\circ} \mathrm{C}$ for $3 \mathrm{~h}$, and reloading to a total strain of 0.045 ; and (iii) loading to a strain of 0.045 with no annealing. The uniaxial stresses reached at a strain of 0.045 were $\sim 100 \mathrm{MPa}$. After the initial strain of 0.025, twins were observed as shown in Figs. 6.a and c. Upon reloading of the non-annealed sample, the growth of existing twins and nucleation and growth of new twins was observed (Fig. 6.b) and the stress-strain curve was nearly identical to samples that were loaded continuously to 0.045. In the annealed sample, however, existing twins did not grow and only new twins were nucleated (Fig. 6.d) with plastic straining resumed at a uniaxial stress $\sim 20 \mathrm{MPa}$ above the value attained upon the initial loading to 0.025 strain. HAADAF-STEM images confirmed that the annealed specimens have strong segregation of $\mathrm{Gd}$ to the twin boundary sites 2 while equilibrium segregation in $\mathrm{Gd}$ for a bulk composition of $c=0.2 \mathrm{at} . \%$, at $150{ }^{\circ} \mathrm{C}$ is predicted by Eq. 31 to be $c_{2}=0.76$, $c_{6}=0.01$, and $c_{1}=c_{5}=0$. From Eq. 41, we compute that a stress of 2.1 $\mathrm{GPa}$ is required to unpin the solute-segregated twin boundaries. Thus, for the system studied by Nie et al., we predict that the segregated twin boundaries will never grow, consistent with the experiments. 

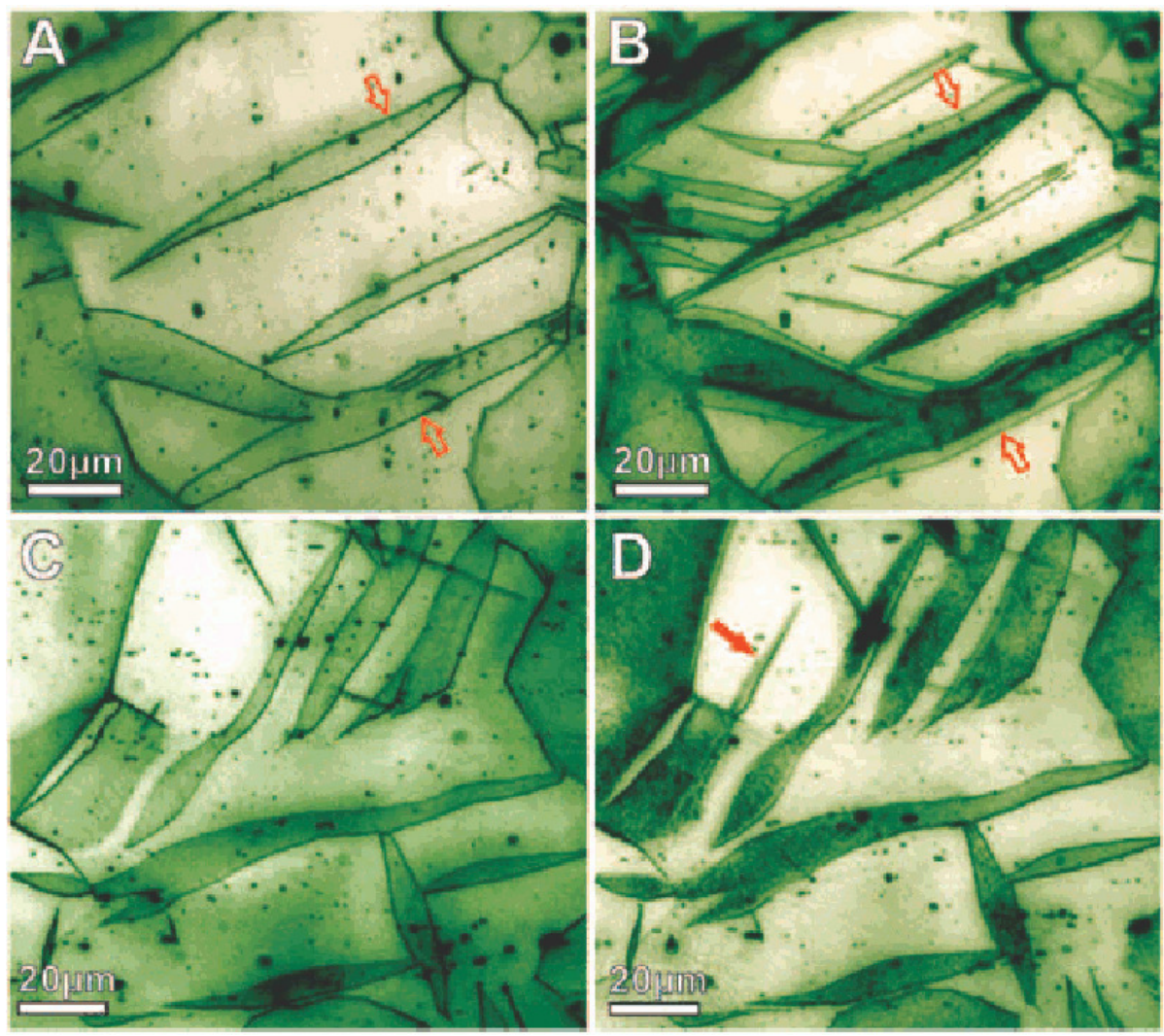

Figure 6: Optical micrographs showing twins in Mg-0.2at.\%Gd alloy. (a) Sample compressed to a strain of 0.025 , and (b) unloaded and immediately recompressed to an accumulated strain of 0.045. (c) Sample compressed to a strain of 0.025 and (d) unloaded, immediately annealed at $150{ }^{\circ} \mathrm{C}$ for $3 \mathrm{~h}$, and recompressed to an accumulated strain of 0.045 [31]. Figure reproduced with permission.

\subsection{Other implications: detwinning and dynamic strain aging}

Detwinning is the narrowing or dissappearing of existing twins as a consequence of unloading or reverse loading, at lower stresses than those required to activate twinning since the heterogeneous nucleation of twins is not involved [40,41]. Our model provides a mechanism for detwinning, since the nucleation process can occur in either direction (twinning or detwinning; only defined by identifying the parent grain) and is only biased by the resolved shear stress acting on the twin boundary. Expansion of a nucleated detwinning loop requires an appropriate resolved shear stress. However, the nucleation process is very local, over the nanometer scale of $L^{* 2}$, and so local stress concentrations can drive local nucleation of detwinning, assisted by appropriate solute fluctuations, evenif the global stresses are such as to drive twinning. Expansion of such a nucleated detwinning loop proceeds until the loop enters a region of reduced driving force (lower resolved shear stress of the appropriate sign). This mechanism can thus 
qualitatively explain the unusual twin morphologies such as those in Figs. 6.a-c (no segregation) where the typical lenticular twins sometimes evolve into irregular shapes. In a forthcoming paper, we will present a model for understanding the lenticular shape. Here we note that the irregular shapes can only be achieved by locally-enhanced twinning or by locally-driven detwinning. Such local stress concentrations could be associated with twin-twin interactions, twin-dislocation interactions, or twin-slip-band interactions.

Dynamic strain aging (DSA) is normally associated with strengthening of a material due to the diffusion of solutes around a dislocation during plastic flow. Recent models demonstrate the aging due to "cross-core" diffusion, in which solutes in the immediate core of the dislocation migrate from the tension to compression side of the core (or vice versa) during periods when the dislocation are pinned by obstacles $[42,43]$. The main observable consequence of DSA is the occurence of serrated flow and Portevin-LeChatelier instabilities, caused when DSA renders the steady-state rate sensitivity of the material negative. Serrated flow has been experimentally observed in various $\mathrm{Mg}$ alloys $[44,45]$ and attributed to DSA, but the mechanisms remain unknown. DSA could occur due to the cross-core mechanism operating on lattice dislocations, but the occurence of DSA in materials with very low solute concentrations suggests that this mechanism cannot achieve the strength levels need to generate the magnitude of observed stress drops (5-10 MPa, depending on the $\mathrm{Mg}$ alloy $[44,46,47])$. Our model of the inhibition of twinning due to solute segregation in the twin boundary provides a potential mechanism for DSA effects in Mg alloys, as follows. First, we note that there is a large difference in solute/twin interaction energies between sites 1 and 2 for all solutes studied to date (Al, Zn and Gd, see Fig. 1). This large difference in energy between two neighboring sites provides a strong thermodynamic driving force and, correspondingly, a lower migration energy, for solute diffusion from the energetically unfavorable site to the energetically favorable site, which we term "within-twin" diffusion. For instance, in Gd, diffusion of a single solute from a site 1 to a neighboring site 2 lowers the solute energy by $0.465 \mathrm{eV}$, which is extremely large. The migration barrier for vacancy-mediated diffusion from site 1 to site 2 is reduced by a comparable amount, and thus this local "within-twin" diffusion is much faster than bulk solute diffusion. The existence of a large thermodynamic driving force and a large reduction in migration barrier for sites in the "core" of a defect is a common feature between within-twin and cross-core diffusion mechanisms. At low plastic shear strain rates, twin growth is also slow and occurring due to individual nucleation and growth events separated by quiescent periods along individual twin boundaries. During the waiting time for the next nucleation event, solute diffusion can occur between sites 1 and 2 at a rate much faster than any bulk diffusion. This diffusion is akin to solute segregation, but occurring only locally in the twin boundary. Considering the limiting case of full "within-twin" segregation, we can use Eq. 41 to estimate the pinning stresses as follows. For Gd, we envision that the "within-twin" segregation drives the system from the random state $c_{1}=c_{2}=c_{5}=c_{6}=c$ to the segregated state $c_{1}=0, c_{2}=2 c, c_{5}=c_{6}=c$ with a corresponding pinning stress $\tau_{\text {pin }}=2270 c$ $\mathrm{MPa}$. For the $0.2 \% \mathrm{Gd}$ magnesium alloy, this pinning stress would be $\sim 4.5 \mathrm{MPa}$. After an increase in applied stress to unpin such segregated boundaries, subsequent growth would occur more rapidly at the elevated applied stress until further hardening occurs, which could be manifested macroscopically as DSA 
and/or serrated flow. There are more details that must be studied to determine if our proposed "within-twin" diffusion can yield DSA, but some key elements can be quantified using the ideas and model presented here.

\section{Conclusions}

A mechanistic model for the nucleation of tensile twins from existing twin boundaries has been developed, validated by molecular dynamics simulations, and applied to evaluate a range of phenomena occuring in $\mathrm{Mg}$ alloys. The model is a mechanism for twin-loop formation, which is absent in existing twin growth models and different from recent shear-coupled-migration models of grain boundary motion. The analysis is based on a robust energy method that accounts for interface energy changes due to solute/twin interactions and solute fluctuations and segregation.

For random solute distributions, the model predicts that twin growth can occur via this mechanism because of typical rare solute fluctuations that can occur somewhere across the large twin area, leading to twin nucleation growth at very low stresses. This implies that twin growth is controlled not by the nucleation of twin loops, but by their expansion, which is associated with more-traditional solute-strengthening of an existing (nucleated) twin dislocation. However, twin nucleation remains a critical necessary initial step for activating plastic strain by twinning. Testing the model against molecular dynamics simulations requires several additional features associated with the finite size of MD models, but excellent agreement is achieved, and twin growth is directly observed at low shear stresses (down to $15 \mathrm{MPa}$ ) during MD time scales for moderate solute concentrations. For segregated solutes, the model predicts significant pinning of twin boundaries, i.e. high stresses are needed to overcome the energy associated with solute segregation to low-energy sites around the twin boundary. These predictions were quantitatively confirmed by MD simulations, and the pinning phenomenon is consistent with recent experiments. Due to the small stresses required for twin loop nucleation, this mechanism is also a possible mechanism for detwinning and, therefore, for rationalizing the complex twin shapes often observed. We also suggest that segregation via "within-twin" diffusion, between sites 1 and 2, may be a mechanism for observed dynamic strain aging in $\mathrm{Mg}$ alloys.

Overall, the new model has a number of attractive physical features, with a particular emphasis on the important role of solutes on the thermally-activated stress-driven nucleation of twins. In addition, the model is not limited to twin boundaries. It may be that the phenomenon occurs at relatively low stresses in twins because the line energy cost for loop nucleation is expected to scale with the Burgers vector and the twin Burgers vector is very small. However, the phenomenon may apply to other grain boundaries, and preliminary MD simulations on other symmetric tilt boundaries in Mg suggest that migration at low stresses and long times can be due to the nucleation and growth mechanism proposed here. Extension of the present model to more-fully address detwinning, dynamic strain aging, and broader applications beyond twin boundaries, are subjects for future investigation. 


\section{Acknowledgments}

Support for this work was provided through a European Research Council Advanced Grant, "Predictive Computational Metallurgy", ERC Grant agreement No. 339081 - PreCoMet and by EPFL through its support for the Laboratory for Multiscale Mechanics Modelling (LAMMM). M. G. acknowledges support for this work through the NSF-GOALI Grant No. 1309687.

\section{Appendix A}

Obtaining of the largest probable $\left\{\left|\alpha_{i}\right|\right\}$ that maximizes the growth rate, requires the maximization of $|\Delta \Gamma|$ subject to the condition $P\left(\left\{\left|\alpha_{i}\right|\right\}\right) N_{\text {tot }}=1$, where

$$
\begin{aligned}
|\Delta \Gamma| & =\frac{\left|E_{5}-E_{1}\right|}{\sqrt{2 \Sigma}}\left[\left|\alpha_{1}\right| \sqrt{c_{1}\left(1-c_{1}\right)}+\left|\alpha_{5}\right| \sqrt{c_{5}\left(1-c_{5}\right)}\right] \\
& +\frac{\left|E_{6}-E_{2}\right|}{\sqrt{2 \Sigma}}\left[\left|\alpha_{2}\right| \sqrt{c_{2}\left(1-c_{2}\right)}+\left|\alpha_{6}\right| \sqrt{c_{6}\left(1-c_{6}\right)}\right]
\end{aligned}
$$

and $P$ is the probability of finding the corresponding fluctuations $\left\{\left|\alpha_{i}\right|\right\}$ over the total number of possible nucleation sites $N_{t o t}$. Since these fluctuations are independent random variables, $P$ can be expressed as

$$
P\left(\left\{\left|\alpha_{i}\right|\right\}\right)=\prod_{i} p\left(\left|x_{i}\right|>\left|\tilde{\alpha}_{i}\right|\right)
$$

where

$$
p\left(\left|x_{i}\right|>\left|\tilde{\alpha}_{i}\right|\right)=\int_{\left|\tilde{\alpha}_{i}\right|}^{\infty} \frac{d x}{\sqrt{2 \pi}} \exp \left(-\frac{x^{2}}{2}\right)
$$

Calling $\alpha_{i}$ the median of the interval $\left(\tilde{\alpha}_{i}, \infty\right)$, verifying $p\left(\left|\tilde{\alpha}_{i}\right|<\left|x_{i}\right|<\left|\alpha_{i}\right|\right)=$ $p\left(\left|x_{i}\right|>\left|\alpha_{i}\right|\right)$, we can easily show that $p\left(\left|x_{i}\right|>\left|\tilde{\alpha}_{i}\right|\right)=2 p\left(\left|x_{i}\right|>\left|\alpha_{i}\right|\right)$. Then $P$ can also be expressed as

$$
P\left(\left\{\left|\alpha_{i}\right|\right\}\right)=\prod_{i} 2 p\left(\left|x_{i}\right|>\left|\alpha_{i}\right|\right)
$$

The maximization of $|\Delta \Gamma|$ is the same as maximizing

$$
\begin{aligned}
& \left|E_{5}-E_{1}\right|\left[\left|\alpha_{1}\right| \sqrt{c_{1}\left(1-c_{1}\right)}+\left|\alpha_{5}\right| \sqrt{c_{5}\left(1-c_{5}\right)}\right] \\
& +\left|E_{6}-E_{2}\right|\left[\left|\alpha_{2}\right| \sqrt{c_{2}\left(1-c_{2}\right)}+\left|\alpha_{6}\right| \sqrt{c_{6}\left(1-c_{6}\right)}\right]
\end{aligned}
$$

subject to the condition $P\left(\left\{\left|\alpha_{i}\right|\right\}\right) N_{\text {tot }}=1$, which defines a Lagrangian multiplier problem. Consider now the function

$$
\begin{aligned}
\Phi\left(\left\{\left|\alpha_{i}\right|\right\}, \lambda\right)= & \left|E_{5}-E_{1}\right|\left[\left|\alpha_{1}\right| \sqrt{c_{1}\left(1-c_{1}\right)}+\left|\alpha_{5}\right| \sqrt{c_{5}\left(1-c_{5}\right)}\right] \\
& +\left|E_{6}-E_{2}\right|\left[\left|\alpha_{2}\right| \sqrt{c_{2}\left(1-c_{2}\right)}+\left|\alpha_{6}\right| \sqrt{c_{6}\left(1-c_{6}\right)}\right] \\
& +\lambda\left(P\left(\left\{\left|\alpha_{i}\right|\right\}\right) N_{t o t}-1\right)
\end{aligned}
$$


being $\lambda$ the unknown Lagrangian multiplier. The solution of this problem is then obtained from the maximization of $\Phi$. For site 1, we then have

$$
\begin{aligned}
\frac{\partial \Phi}{\partial\left|\alpha_{1}\right|} & =0 \\
& =\left|E_{5}-E_{1}\right| \sqrt{c_{1}\left(1-c_{1}\right)}+\lambda N_{t o t} \frac{\partial P}{\partial\left|\alpha_{1}\right|} \\
& =\left|E_{5}-E_{1}\right| \sqrt{c_{1}\left(1-c_{1}\right)}+2 \lambda N_{t o t} \frac{d p\left(\left|x_{1}\right|>\left|\alpha_{1}\right|\right)}{d\left|\alpha_{1}\right|} \prod_{j \neq 1} 2 p\left(\left|x_{j}\right|>\left|\alpha_{j}\right|\right) \\
& =\left|E_{5}-E_{1}\right| \sqrt{c_{1}\left(1-c_{1}\right)}-\lambda N_{t o t} \sqrt{\frac{2}{\pi}} \exp \left(-\frac{\alpha_{1}^{2}}{2}\right) \prod_{j \neq 1} 2 p\left(\left|x_{j}\right|>\left|\alpha_{j}\right|\right)
\end{aligned}
$$

In the asymptotic limit given by $\left|\alpha_{i}\right| \gg 1$,

$$
p\left(\left|x_{i}\right|>\left|\alpha_{i}\right|\right) \sim \frac{1}{\sqrt{2 \pi}} \frac{1}{\left|\alpha_{i}\right|} \exp \left(-\frac{\alpha_{i}^{2}}{2}\right)
$$

then

$$
\frac{1}{\sqrt{2 \pi}} \exp \left(-\frac{\alpha_{i}^{2}}{2}\right) \sim\left|\alpha_{i}\right| p\left(\left|x_{i}\right|>\left|\alpha_{i}\right|\right)
$$

Substituting this relation for $i=1$ in the above expression and recalling that $P N_{t o t}=1$, then

$$
\left|E_{5}-E_{1}\right| \sqrt{c_{1}\left(1-c_{1}\right)}-\lambda\left|\alpha_{i}\right|=0
$$

Solving for $\left|\alpha_{1}\right|$, since $\lambda \neq 0$, we get

$$
\left|\alpha_{1}\right|=\frac{\left|E_{5}-E_{1}\right| \sqrt{c_{1}\left(1-c_{1}\right)}}{\lambda}
$$

Proceeding similarly for sites 2,5 and 6 , we get

$$
\begin{aligned}
& \left|\alpha_{2}\right|=\frac{\left|E_{6}-E_{2}\right| \sqrt{c_{2}\left(1-c_{2}\right)}}{\lambda} \\
& \left|\alpha_{5}\right|=\frac{\left|E_{5}-E_{1}\right| \sqrt{c_{5}\left(1-c_{5}\right)}}{\lambda} \\
& \left|\alpha_{6}\right|=\frac{\left|E_{6}-E_{2}\right| \sqrt{c_{6}\left(1-c_{6}\right)}}{\lambda}
\end{aligned}
$$


Once the values of $\left\{\left|\alpha_{i}\right|\right\}$ are expressed in terms of $\lambda, P\left(\left\{\left|\alpha_{i}\right|\right\}\right) N_{\text {tot }}=1$ can be solved for $\lambda$. In the asymptotic limit,

$$
\begin{aligned}
1= & N_{t o t} \prod_{i} 2 p\left(\left|x_{i}\right|>\left|\alpha_{i}\right|\right)=\frac{4 N_{t o t}}{\pi^{2}} \frac{1}{\prod_{i}\left|\alpha_{i}\right|} \exp \left(-\frac{1}{2} \sum_{i} \alpha_{i}^{2}\right) \\
= & \frac{4 N_{t o t}}{\pi^{2}} \frac{\lambda^{4}}{\left(E_{5}-E_{1}\right)^{2}\left(E_{6}-E_{2}\right)^{2} \prod_{i} \sqrt{c_{i}\left(1-c_{i}\right)}} \\
& \times \exp \left(-\frac{1}{2 \lambda^{2}}\left[\left(E_{5}-E_{1}\right)^{2}\left(c_{1}\left(1-c_{1}\right)+c_{5}\left(1-c_{5}\right)\right)\right.\right. \\
& \left.\left.+\left(E_{6}-E_{2}\right)^{2}\left(c_{2}\left(1-c_{2}\right)+c_{6}\left(1-c_{6}\right)\right)\right]\right)
\end{aligned}
$$

Since the exponential term dominates,

$$
\begin{aligned}
\lambda \sim\left\{\frac{1}{2}[\right. & \left.\ln \left(\frac{4 N_{t o t}}{\pi^{2}}\right)\right]^{-1}\left[\left(E_{5}-E_{1}\right)^{2}\left(c_{1}\left(1-c_{1}\right)+c_{5}\left(1-c_{5}\right)\right)\right. \\
& \left.\left.+\left(E_{6}-E_{2}\right)^{2}\left(c_{2}\left(1-c_{2}\right)+c_{6}\left(1-c_{6}\right)\right)\right]\right\}^{1 / 2}
\end{aligned}
$$

Substitution of $\lambda$ into $\left\{\left|\alpha_{i}\right|\right\}$ yields the following general result

$$
\begin{aligned}
\left|\alpha_{1}\right| \sim & \left\{2 \operatorname { l n } ( \frac { 4 N _ { t o t } } { \pi ^ { 2 } } ) c _ { 1 } ( 1 - c _ { 1 } ) \left[\left(c_{1}\left(1-c_{1}\right)+c_{5}\left(1-c_{5}\right)\right)\right.\right. \\
& \left.\left.+\left(\frac{E_{6}-E_{2}}{E_{5}-E_{1}}\right)^{2}\left(c_{2}\left(1-c_{2}\right)+c_{6}\left(1-c_{6}\right)\right)\right]^{-1}\right\}^{1 / 2} \\
\left|\alpha_{2}\right| \sim \mid & \left|\frac{E_{6}-E_{2}}{E_{5}-E_{1}}\right|\left\{2 \operatorname { l n } ( \frac { 4 N _ { t o t } } { \pi ^ { 2 } } ) c _ { 2 } ( 1 - c _ { 2 } ) \left[\left(c_{1}\left(1-c_{1}\right)+c_{5}\left(1-c_{5}\right)\right)\right.\right. \\
& \left.\left.+\left(\frac{E_{6}-E_{2}}{E_{5}-E_{1}}\right)^{2}\left(c_{2}\left(1-c_{2}\right)+c_{6}\left(1-c_{6}\right)\right)\right]^{-1}\right\}^{1 / 2} \\
\left|\alpha_{5}\right| \sim & \left\{2 \operatorname { l n } ( \frac { 4 N _ { t o t } } { \pi ^ { 2 } } ) ^ { 2 } c _ { 5 } ( 1 - c _ { 5 } ) \left[\left(c_{1}\left(1-c_{1}\right)+c_{5}\left(1-c_{5}\right)\right)\right.\right. \\
& \left.\left.+\left(\frac{E_{6}-E_{2}}{E_{5}-E_{1}}\right)^{2}\left(c_{2}\left(1-c_{2}\right)+c_{6}\left(1-c_{6}\right)\right)\right]^{-1}\right\}^{1 / 2}
\end{aligned}
$$


and

$$
\begin{gathered}
\left|\alpha_{6}\right| \sim\left|\frac{E_{6}-E_{2}}{E_{5}-E_{1}}\right|\left\{2 \operatorname { l n } ( \frac { 4 N _ { t o t } } { \pi ^ { 2 } } ) c _ { 6 } ( 1 - c _ { 6 } ) \left[\left(c_{1}\left(1-c_{1}\right)+c_{5}\left(1-c_{5}\right)\right)\right.\right. \\
\left.\left.+\left(\frac{E_{6}-E_{2}}{E_{5}-E_{1}}\right)^{2}\left(c_{2}\left(1-c_{2}\right)+c_{6}\left(1-c_{6}\right)\right)\right]^{-1}\right\}^{1 / 2}
\end{gathered}
$$

If we now take the accurate approximation of $\left|E_{5}-E_{1}\right| \approx\left|E_{6}-E_{2}\right|$, we then get

$$
\left|\alpha_{i}\right| \sim \sqrt{2 \ln \left(\frac{4 N_{t o t}}{\pi^{2}}\right) \frac{c_{i}\left(1-c_{i}\right)}{\sum_{j} c_{j}\left(1-c_{j}\right)}}
$$

which is the result given in the text. Should any of the sites present $c_{i}=0$ or $c_{i}=1$, the associated $\alpha_{i}$ would be 0 , which does not satisfy the $\left|\alpha_{i}\right| \gg 1$ condition. Nevertheless, Eq. A.15 yields the right value of $\left|\alpha_{i}\right|$ in that situation, even if the expression was obtained considering the asymptotic limit for all sites.

\section{References}

[1] Suwas S, Gottstein G, Kumar R. Mater. Sci. Eng. A 2007;471:1.

[2] Mackenzie L W F, Pekguleryuz M. Mater. Sci. Eng. A 2008;480:189.

[3] Elsayed A, Umeda J, Kondoh K. Materials and Design 2011;32:4590.

[4] Morris J R, Ye Y, Yoo M H. Philo. Mag. 2003;85.233.

[5] Han J, Su X M, Jin Z H, Zhu Y T. Scripta Mater. 2011;64:693.

[6] Lin L, Wang F, Yang L, Chen L J, Liu Z, Wang Y M. Mater. Sci. Eng. A 2011;528:5283.

[7] Leyson G P M, Hector Jr L G, Curtin W A. Acta Mat. 2012;60:5197.

[8] Wang J, Hirth J P, Tomé C N. Acta Mat. 2009;57:5521.

[9] Nogaret T, Curtin W A, Yasi J A, Hector Jr L G, Trinkle D R. Acta Mat. 2010;58:4332.

[10] Song H Y, Li Y L. Phys. Lett. A 2012;376:529.

[11] Beausir B, Suwas S, Tóth L S, Neale K W, Fundenberger J J. Acta Mat. 2008;56:200.

[12] Mayama T, Aizawa K, Todano Y, Kuroda M. Comp. Mat. Sci. 2009;47:448.

[13] Wang H, Wu P D, Tomé C N, Wang J. Int. J. Solids Struct. 2012;49:2155.

[14] Hirth J P, Lothe J. Theory of dislocations. New York: Wiley; 1982.

[15] Thompson N, Millard D J. Philo. Mag. 1952;43:422.

[16] Serra A, Bacon D J, Pond R C Acta Metall. 1988;36:3183. 
[17] Luque A, Ghazisaeidi M, Curtin W A. Modelling Simul. Mater. Sci. Eng. 2013;21:045010.

[18] Beyerlein I J, McCabe R J, Tomé C N. J. Mech. Phys. Solids 2011;59:988.

[19] Barnett M, Setty M, Siska F. Metall. Mater. Trans. A 2012;44A:2962.

[20] Yuasa M, Masunaga K, Yoshida T, Mabuchi M, Chino Y. Acta Mat. 2013;61:4714.

[21] Zhou N, Zhang Z, Jin L, Dong J, Chen B, Ding W. Materials and Design 2014;56:966.

[22] El Kadiri H, Kapil J, Oppedal A L, Hector Jr L G, Agnew S R, Cherkaoui M, Vogel S C. Acta Mat. 2013;61:3549.

[23] Barrett C D, El Kadiri H, Tschopp M A. J. Mech. Phys. Solids 2012;60:2084.

[24] Su J, Kaboli S, Kabir A S H, Jung I-H, Yue S. Mater. Sci. Eng. A 2013;587:27.

[25] Cahn J W, Mishin Y, Suzuki A. Acta Mat. 2006;54:4953.

[26] Luque A, Aldazabal J, Martínez-Esnaola J M, Gil Sevillano J. Philo. Mag. 2010;90:3743.

[27] Wang J, Beyerlein I J. Modelling Simul. Mater. Sci. Eng. 2012;20:024002.

[28] Wang J, Beyerlein I J, Tomé C N. Int. J. Plasticity 2014;56:156.

[29] Ghazisaeidi M, Hector Jr L G, Curtin W A. Under revision.

[30] Ghazisaeidi M, Curtin W A. Modelling Simul. Mat. Sci. Eng. 2013;21:055007.

[31] Nie J F, Zhu Y M, Liu J Z, Fang X Y. Science 2013;340:957.

[32] Liu X-Y, Ohotnicky P P, Adams J B, Lane Rohrer C, Hyland Jr R W. Surf. Sci. 1997;373:357.

[33] Stukowski A. Modelling Simul. Mater. Sci. Eng. 2010;18:015012.

[34] Reif F. Fundamentals of statistical and thermal physics. New York: McGraw-Hill; 1965.

[35] Plimpton S. J. Comp. Phys. 1995;117:1.

[36] Kittel C, Kroemer H. Thermal Physics. New York: Freeman; 1980.

[37] Kelley E N, Hosford W F. Trans. Metall. Soc. of AIME 1968;242:5.

[38] Chapuis A, Driver J H. Acta Mat. 2011;59:1986.

[39] Stanford N, Barnett M R. Int. J. Plasticity 2013;47:165.

[40] Lou X Y, Li M, Boger R K, Agnew S R, Wagoner R H. Int. J. Plasticity 2007;23:44. 
[41] Sarker D, Chen D L. Scripta Mater. 2012;67:165.

[42] Curtin W A, Olmsted D L, Hector Jr L G. Nature Materials 2006;5:875.

[43] Soare M A, Curtin W A. Acta Mat. 2008;56:4046.

[44] Stanford N, Sabirov I, Sha G, La Fontaine A, Ringer S P, Barnett M R. Metallurgical and Materials Transactions A 2010;41A:734.

[45] Stanford N, Sha G, Xia J H, Ringer S P, Barnett M R. Scripta Mater. 2011;65:919.

[46] Jiang L, Jonas J J, Mishra R. Mater. Sci. Eng. A 2011;528:6596.

[47] Dudamell N V, Hidalgo-Manrique P, Chakkedath A, Chen Z, Boehlert C J, Gálvez F, Yi S, Bohlen J, Letzig D, Pérez-Prado M T. Mater. Sci. Eng. A 2013;583:220. 